SUPPORTING INFORMATION

for

\title{
New synthesis of semisquaric acid derivatives via chlorinated $N$-(cyclobutylidene)amines
}

\author{
Guido Verniest, Jeroen Colpaert, Karl W. Törnroos ${ }^{\S}$ and Norbert De Kimpe* \\ Department of Organic Chemistry, Faculty of Agricultural and Applied Biological Sciences, \\ Ghent University, Coupure Links 653, 9000 Ghent, Belgium
}

CONTENTS:

General methods

Synthetic procedures and ${ }^{1} \mathrm{H},{ }^{13} \mathrm{C}$, IR, MS and El. Anal. data of compounds 12, 13, 14, 15, 16, 17, 18, 20, 21, 29 and 30.

X-ray analytical data for compound 29a.

$(\mathrm{S} 16-\mathrm{S} 18)$

Copies of ${ }^{1} \mathrm{H}$ and ${ }^{13} \mathrm{C}$ spectra of known compounds $1, \mathbf{1 7} \mathbf{a}, \mathbf{1 7} \mathbf{b}, \mathbf{1 7} \mathbf{c}, \mathbf{1 7 d}, \mathbf{3 0 e} \quad$ (S19-S30)

Copies of ${ }^{1} \mathrm{H}$ and ${ }^{13} \mathrm{C}$ spectra of new compounds 12e, 20d, 20e, 21d, 21e

(S31-S40)

$\S$ Department of Chemistry, University of Bergen, Allegaten 41, 5007 Bergen, Norway

" Corresponding author. Tel.: +32 (0)9 26459 50; fax: +32 (0)9 26462 43; e-mail: norbert.dekimpe@UGent.be. 


\section{General methods.}

${ }^{1} \mathrm{H}$ NMR and ${ }^{13} \mathrm{C}$ NMR spectra were run at $270 \mathrm{MHz}$ or $300 \mathrm{MHz}$ and $68 \mathrm{MHz}$ or $75 \mathrm{MHz}$, respectively. The compounds were diluted in deuterated solvents $\left(\mathrm{CDCl}_{3}, \mathrm{D}_{2} \mathrm{O}\right.$, acetone- $\mathrm{d}_{6}$ or DMSO- $\left.\mathrm{d}_{6}\right)$ with tetramethylsilane $(\mathrm{TMS})$ as reference $(\delta=0 \mathrm{ppm})$. Mass spectra were recorded using either GC-MS (70eV) coupling or a direct inlet system. LC-MS was performed using a positive or negative electrospray.

\section{Synthetic procedures and spectral data}

\section{Synthesis of imines 12}

$N$-(3-Phenylcyclobutylidene)isopropylamine (12a). To a solution of $30.00 \mathrm{~g}$ (205.48

mmol) 3-phenylcyclobutanone $11 a^{27}$ in $200 \mathrm{ml}$ of dry diethyl ether was added $48.50 \mathrm{~g}$ (821.92 mmol, 4 equiv.) isopropylamine. The mixture was cooled to $0^{\circ} \mathrm{C}$ and subsequently, $23.41 \mathrm{~g}$ (123.29 mmol, 0.6 equiv.) titanium(IV) chloride in $25 \mathrm{ml}$ of dry petroleum ether was added dropwise during 30 minutes. After reaction at $0^{\circ} \mathrm{C}$ for 1 hour, the ice bath was removed and the mixture was allowed to reach room temperature and stirring was continued overnight. After reaction, the milky mixture was poured in $200 \mathrm{ml}$ of $0.5 \mathrm{M} \mathrm{NaOH}$ and rapidly extracted with diethyl ether $(3 \times 200 \mathrm{ml})$. The combined extracts were dried over a mixture of $\mathrm{K}_{2} \mathrm{CO}_{3}$ and $\mathrm{MgSO}_{4}$ and the solvent evaporated at room temperature. The resulting imine 12a was purified by distillation. Bp: $68-75{ }^{\circ} \mathrm{C}, 0.2 \mathrm{mmHg}$; yield $81 \% .{ }^{1} \mathrm{H} \mathrm{NMR}\left(300 \mathrm{MHz}, \mathrm{CDCl}_{3}\right) \delta$ $1.14(3 \mathrm{H}, \mathrm{d}, J=6.3 \mathrm{~Hz}), 1.15(3 \mathrm{H}, \mathrm{d}, J=6.3 \mathrm{~Hz}), 2.89-3.11(2 \mathrm{H}, \mathrm{m}), 3.28-3.55(4 \mathrm{H}, \mathrm{m}), 7.17-$ $7.34(5 \mathrm{H}, \mathrm{m}) ;{ }^{13} \mathrm{C}$ NMR $\left(75 \mathrm{MHz}, \mathrm{CDCl}_{3}\right) \delta 23.6,23.7,31.8,41.8,45.3,52.0,126.3,2 \times$ 
126.4, $2 \times 128.5,144.7,165.4 ; \mathrm{IR}(\mathrm{NaCl}) v_{\max } 1710,1603,1495,1455 \mathrm{~cm}^{-1} ; \mathrm{MS} \mathrm{m} / z(\%): 187$ $\left(\mathrm{M}^{+}, 14\right), 131$ (10), $104(20), 83$ (100), 55 (27), 43 (48), 41 (92); Anal. Calcd. for $\mathrm{C}_{13} \mathrm{H}_{17} \mathrm{~N}$ : C, 83.37; H, 9.15; N, 7.48. Found: C, 83.11; H, 9.27; N, 7.35.

$N$-(3-Phenylcyclobutylidene)-t-butylamine 12b. (No purification, unstable compound) ${ }^{1} \mathrm{H}$ NMR (270 MHz, $\left.\mathrm{CDCl}_{3}\right) \delta 1.25(9 \mathrm{H}, \mathrm{s}), 3.05-3.20$ and 3.25-3.60 (5H, m), 7.20-7.35 (5H, m); ${ }^{13} \mathrm{C}$ NMR $\left(68 \mathrm{MHz}, \mathrm{CDCl}_{3}\right) \delta 3 \times 30.0,32.2,46.4,47.3,56.4,126.3,2 \times$ 126.5, $2 \times 128.5,145.0,164.9 ; \mathrm{IR}(\mathrm{NaCl}) v_{\max } 1705,1600,1491,1452 \mathrm{~cm}^{-1} ; \mathrm{MS} \mathrm{m} / z(\%): 201$ $\left(\mathrm{M}^{+}, 10\right), 186(29), 144(31), 57$ (100), 44 (24), 4139$)$.

$N$-(3-Phenylcyclobutylidene)cyclohexylamine 12c. (No purification, unstable compound) ${ }^{1} \mathrm{H}$ NMR $\left(270 \mathrm{MHz}, \mathrm{CDCl}_{3}\right) \delta 1.23-1.83(10 \mathrm{H}, \mathrm{m}), 2.90-3.16(3 \mathrm{H}, \mathrm{m}), 3.29-3.45$ $(2 \mathrm{H}, \mathrm{m}), 3.51-3.63(1 \mathrm{H}, \mathrm{m}), 7.18-7.42(5 \mathrm{H}, \mathrm{m}) ;{ }^{13} \mathrm{C} \mathrm{NMR}\left(68 \mathrm{MHz}, \mathrm{CDCl}_{3}\right) \delta 2 \times 24.2,25.1$, $32.2,2 \times 33.2,41.0,44.9,59.8,125.7,2 \times 125.8,2 \times 127.9,144.2,165.3 ; \mathrm{IR}(\mathrm{NaCl}) v_{\max }$ 1709, 1603, 1495, $1449 \mathrm{~cm}^{-1}$; MS (ES+) $\mathrm{m} / z(\%): 228\left(\mathrm{M}+\mathrm{H}^{+}, 100\right)$.

$N$-(3-(4-Chlorophenyl)cyclobutylidene)isopropylamine $12 \mathrm{~d}$. Distillation $\left(93^{\circ}-98^{\circ} \mathrm{C}\right.$, $0.01 \mathrm{mmHg}) .{ }^{1} \mathrm{H}$ NMR $\left(300 \mathrm{MHz}, \mathrm{CDCl}_{3}\right) \delta 1.14(3 \mathrm{H}, \mathrm{d}, J=6.3 \mathrm{~Hz}), 1.15(3 \mathrm{H}, \mathrm{d}, J=6.3 \mathrm{~Hz})$, 2.85-3.07 (2H, m), 3.29-3.61 (4H, m), $7.20(2 \mathrm{H}, \mathrm{d}, J=8.3 \mathrm{~Hz}), 7.29(2 \mathrm{H}, \mathrm{d}, J=8.3 \mathrm{~Hz}) ;{ }^{13} \mathrm{C}$ NMR $\left(75 \mathrm{MHz}, \mathrm{CDCl}_{3}\right) \delta 23.0,23.1,30.7,41.3,44.7,51.4,2 \times 127.2,2 \times 127.9,131.3$, 142.6, 164.3; IR (NaCl): $v_{\max } 1710,1493 \mathrm{~cm}^{-1} . \mathrm{MS} \mathrm{m} / \mathrm{z}(\%): 221 / 23\left(\mathrm{M}^{+}, 2\right), 138 / 40(40), 83$ (100); Anal. Calcd. for $\mathrm{C}_{13} \mathrm{H}_{16} \mathrm{NCl}$ C, 70.42; H, 7.27; N, 6.32. Found: C, 70.03; H, 7.34; N, 6.28 .

$N$-(3-Butylcyclobutylidene)-t-butylamine 12e. Distillation $\left(37^{\circ} \mathrm{C}, 0.05 \mathrm{mmHg}\right) .{ }^{1} \mathrm{H}$ $\operatorname{NMR}\left(270 \mathrm{MHz}, \mathrm{CDCl}_{3}\right) \delta 0.90(3 \mathrm{H}, \mathrm{t}, J=6.9 \mathrm{~Hz}), 1.10(6 \mathrm{H}, \mathrm{d}, J=6.3 \mathrm{~Hz}), 1.21-1.37(4 \mathrm{H}, \mathrm{m})$, 1.46-1.54 (2H, m), 2.19-2.52 (3H, m), 2.92-3.02 (2H, m), $3.40(1 \mathrm{H}$, sept, $J=6.3 \mathrm{~Hz}) ;{ }^{13} \mathrm{C}$ NMR $\left(68 \mathrm{MHz}, \mathrm{CDCl}_{3}\right) \delta 14.1,22.6,2 \times 23.7,27.4,30.1,36.3,39.6,43.4,51.7,167.4$; IR $(\mathrm{NaCl}): v_{\max } 1709 \mathrm{~cm}^{-1} . \mathrm{MS} \mathrm{m} / \mathrm{z}(\%): 167\left(\mathrm{M}^{+}, 1\right), 83$ (100), 68 (10), 55 (39), 43 (71), $41(41)$. 


\section{Tetrachlorination of imines 12}

$N$-(2,2,4,4-Tetrachloro-3-phenylcyclobutylidene)isopropylamine (13a). To a solution of $6.85 \mathrm{~g}$ (36.63 mmol) $N$-(3-phenylcyclobutylidene)isopropylamine 12a in $250 \mathrm{ml}$ of dry $\mathrm{CCl}_{4}$ was added $22.01 \mathrm{~g}(164.84 \mathrm{mmol}, 4.5$ equiv.) of NCS and the mixture was refluxed for $30 \mathrm{~min}$. When this reaction was performed on larger scale (e.g. $30 \mathrm{~g}$, the NCS was added in portions during reflux over a period of $15 \mathrm{~min}$ (exothermic reaction!) and was subsequently refluxed for $30 \mathrm{~min}$ ). After the reaction was complete, the mixture was cooled in an ice bath and filtered. To remove remaining succinimide, the filtrate was cooled again for 2 hours at $-20^{\circ} \mathrm{C}$ and subsequently filtered. After evaporation of the $\mathrm{CCl}_{4}$, a crystalline compound was obtained in high purity. The last impurities were removed via recrystallization. Recrystallization (EtOAc/hexane 1:5); yield 100\%, mp 83-84 ${ }^{\circ} \mathrm{C} .{ }^{1} \mathrm{H}$ NMR $\left(300 \mathrm{MHz}, \mathrm{CDCl}_{3}\right.$ ) $\delta 1.35(3 \mathrm{H}, \mathrm{d}, J=6.3 \mathrm{~Hz}), 1.38(3 \mathrm{H}, \mathrm{d}, J=6.3 \mathrm{~Hz}), 4.66(1 \mathrm{H}, \mathrm{sept}, J=6.3 \mathrm{~Hz}), 4.88(1 \mathrm{H}, \mathrm{s})$, 7.34-7.49 (3H, m), 7.57-7.64 (2H, m); ${ }^{13} \mathrm{C}$ NMR (75MHz, $\left.\mathrm{CDCl}_{3}\right) \delta$ 22.7, 22.8, 52.2, 71.3, 78.1, 81.4, $2 \times 128.4,128.8,2 \times 129.5,130.2,158.3 . \mathrm{IR}(\mathrm{KBr}): v_{\max } 1706,1500 \mathrm{~cm}^{-1} . \mathrm{MS} \mathrm{m} / \mathrm{z}$ (\%): no $\mathrm{M}^{+}$, 287/89/91/93 (M+-HCl, 18), 245 (100), 183 (31), 43 (68), 41 (39); Anal. Calcd. for $\mathrm{C}_{13} \mathrm{H}_{13} \mathrm{NCl}_{4}$ : C, 48.03; H, 4.03; N, 4.31. Found: C, 48.26; H, 4.11; N, 4.24.

\section{$N$-(2,2,4,4-Tetrachloro-3-phenylcyclobutylidene)-t-butylamine}

$13 b$.

Recrystallization (EtOAc/hexane 1:4); yield 45\%, mp 74-75 ${ }^{\circ} \mathrm{C} .{ }^{1} \mathrm{H} \mathrm{NMR}\left(270 \mathrm{MHz}, \mathrm{CDCl}_{3}\right) \delta$ $1.57(9 \mathrm{H}, \mathrm{s}), 4.86(1 \mathrm{H}, \mathrm{s}), 7.44-7.48(3 \mathrm{H}, \mathrm{m}), 7.61-7.64(2 \mathrm{H}, \mathrm{m}) ;{ }^{13} \mathrm{C} \mathrm{NMR}\left(68 \mathrm{MHz}, \mathrm{CDCl}_{3}\right)$ $\delta 3 \times 30.4,59.1,70.7,80.9,83.1,2 \times 128.2,128.6,2 \times 129.3,130.4,153.0 ; \mathrm{IR}(\mathrm{KBr}): v_{\max }$ 1710, $1500 \mathrm{~cm}^{-1}$. MS m/z (\%): no $\mathrm{M}^{+}, 322 / 24 / 26 / 28 / 30\left(\mathrm{M}^{+}-\mathrm{Me}, 1\right), 246 / 48 / 50 / 52(6), 183 / 5 / 7$ (17), 57 (100); Anal. Calcd. for $\mathrm{C}_{14} \mathrm{H}_{15} \mathrm{NCl}_{4}$ : C, 49.59; H, 4.46; N, 4.13. Found: C, 49.71; H, $4.55 ; \mathrm{N}, 4.02$. 
$N$-(2,2,4,4-Tetrachloro-3-phenylcyclobutylidene)cyclohexylamine 13c. Flash chromatography (EtOAc/hexane 1:9, Rf $=0.39$ ); yield $42 \%, \mathrm{mp} 122-123^{\circ} \mathrm{C} .{ }^{1} \mathrm{H} \mathrm{NMR}$ $\left(270 \mathrm{MHz}, \mathrm{CDCl}_{3}\right) \delta 1.25-1.92(10 \mathrm{H}, \mathrm{m}), 4.24-4.32(1 \mathrm{H}, \mathrm{m}), 4.88(1 \mathrm{H}, \mathrm{s}), 7.43-7.51(3 \mathrm{H}, \mathrm{m})$, 7.58-7.61 (2H, m); ${ }^{13} \mathrm{C}$ NMR $\left(68 \mathrm{MHz}, \mathrm{CDCl}_{3}\right) \delta 2 \times 24.0,25.3,32.4,32.5,60.1,71.2,78.0$, 81.4, $2 \times 128.3,128.7,2 \times 129.4,130.1,158.4 ; \mathrm{IR}(\mathrm{KBr}): v_{\max } 1703,1443 \mathrm{~cm}^{-1} . \mathrm{MS} \mathrm{m} / z(\%):$ no $\mathrm{M}^{+}, 331 / 3 / 5 / 7\left(\mathrm{M}^{+}-\mathrm{HCl}, 1\right), 191 / 3 / 5$ (19), 83 (100); Anal. Calcd. for $\mathrm{C}_{16} \mathrm{H}_{17} \mathrm{NCl}_{4}$ : C, 52.63; H, 4.69; N, 3.84. Found: C, 52.71; H, 4.82; N, 4.02.

$N$-(2,2,4,4-Tetrachloro-3-(4-chlorophenyl)cyclobutylidene)isopropylamine $13 \mathrm{~d}$. Recrystallization (EtOAc/hexane 1:5); yield 98\%, mp $104-105^{\circ} \mathrm{C} .{ }^{1} \mathrm{H}$ NMR $(300 \mathrm{MHz}$, $\left.\mathrm{CDCl}_{3}\right) \delta 1.35(3 \mathrm{H}, \mathrm{d}, J=6.2 \mathrm{~Hz}), 1.38(3 \mathrm{H}, \mathrm{d}, J=6.2 \mathrm{~Hz}), 4.64(1 \mathrm{H}, \mathrm{sept}, J=6.2 \mathrm{~Hz}), 4.83$ $(1 \mathrm{H}, \mathrm{s}), 7.52(2 \mathrm{H}, \mathrm{d}, J=8.7 \mathrm{~Hz}), 7.45(2 \mathrm{H}, \mathrm{d}, J=8.7 \mathrm{~Hz}) .{ }^{13} \mathrm{C} \mathrm{NMR}\left(\mathrm{CDCl}_{3}\right): \delta 22.8,22.9$, $52.4,70.8,2 \times 81.3,128.6,2 \times 128.8,130.9,135.2,158.0 . \mathrm{IR}(\mathrm{KBr}): v_{\max } 1708,1495 \mathrm{~cm}^{-1}$ MS $m / z(\%)$ : no $\mathrm{M}^{+}, 321 / 23 / 25 / 27$ ( $\left.\mathrm{M}^{+}-\mathrm{HCl}, 24\right), 279 / 81 / 83 / 85 / 87$ ( $\left.\mathrm{M}^{+}-i-\mathrm{Pr}, 83\right), 244$ (100), 217 (47). Anal. Calcd. for $\mathrm{C}_{13} \mathrm{H}_{12} \mathrm{NCl}_{5}$ : C, 43.43; H, 3.36; N, 3.90. Found: C, 43.59; H, 3.24; $\mathrm{N}, 4.08$.

$N$-(3-Butyl-2,2,4,4-tetrachlorocyclobutylidene)isopropylamine 13e. Distillation $\left(62-71{ }^{\circ} \mathrm{C}, 0.01 \mathrm{mmHg}\right)$. Yield 84\%. ${ }^{1} \mathrm{H}$ NMR $\left(270 \mathrm{MHz}, \mathrm{CDCl}_{3}\right) \delta 0.90(3 \mathrm{H}, \mathrm{t}, J=6.9 \mathrm{~Hz})$, $1.21(3 \mathrm{H}, \mathrm{d}, J=6.3 \mathrm{~Hz}), 1.24(3 \mathrm{H}, \mathrm{d}, J=6.3 \mathrm{~Hz}), 1.31-1.51(4 \mathrm{H}, \mathrm{m}), 1.81-1.90(2 \mathrm{H}, \mathrm{m}), 3.38$ $(1 \mathrm{H}, \mathrm{m}), 4.46(1 \mathrm{H}$, sept $J=6.3 \mathrm{~Hz}) ;{ }^{13} \mathrm{C} \mathrm{NMR}\left(68 \mathrm{MHz}, \mathrm{CDCl}_{3}\right) \delta 13.8,22.6,2 \times 22.6,27.1$, 28.5, 51.6, 67.8, 78.3, 81.7, 159.0; IR (KBr): $v_{\max } 1712 \mathrm{~cm}^{-1} . \mathrm{MS} m / z(\%):$ no $\mathrm{M}^{+}, 151 / 3 / 5$ (59), 109/11/13 (31), 43 (100); Anal. Calcd. for $\mathrm{C}_{11} \mathrm{H}_{17} \mathrm{NCl}_{4}$ : C, 43.31; H, 5.62; N, 4.59. Found: C, 43.22; H, 5.87; N, 4.50. 


\section{Hydrolysis of imine 13a}

To $1.00 \mathrm{~g}$ (3.08 mmol) of imine $\mathbf{1 3 a}$ was added $10 \mathrm{ml}$ of aqueous $80 \% \mathrm{H}_{2} \mathrm{SO}_{4}$. After stirring for $20 \mathrm{~h}$ at room temperature, the mixture was poured over ice and extracted with $3 \times$ $20 \mathrm{ml}$ of dichloromethane. After drying and evaporation of the solvents, the resulting reaction mixture was chromatographed on silicagel, yielding 2,4,4-trichloro-3-phenylcyclobutenone 15 and $N$-(2,4,4-trichloro-3-phenyl-2-cyclobutenylidene)isopropylamine $\mathbf{1 4}$ in $21 \%$ and $28 \%$ yield respectively.

$N$-(2,4,4-Trichloro-3-phenyl-2-cyclobutenylidene)isopropylamine $14 . \quad$ Flash chromatography (hexane/EtOAc 9:1, $\left.\mathrm{R}_{\mathrm{f}}=0.50\right)$; yield $28 \%, \mathrm{mp} 89-91^{\circ} \mathrm{C} .{ }^{1} \mathrm{H} \mathrm{NMR}(300 \mathrm{MHz}$, $\left.\mathrm{CDCl}_{3}\right) \delta 1.34(6 \mathrm{H}, \mathrm{d}, J=6.3 \mathrm{~Hz}), 4.54(1 \mathrm{H}, \mathrm{sept}, J=6.3 \mathrm{~Hz}), 7.52-7.55(3 \mathrm{H}, \mathrm{m}), 8.01-8.05$ $(2 \mathrm{H}, \mathrm{m}) ;{ }^{13} \mathrm{C} \mathrm{NMR}\left(75 \mathrm{MHz}, \mathrm{CDCl}_{3}\right) \delta 2 \times 23.6,51.6,81.7,126.8,2 \times 128.5,128.8,2 \times 129.3$, 131.9, 154.3, 156.0. IR (KBr): $v_{\max } 1722,1585 \mathrm{~cm}^{-1} . \mathrm{MS}(\mathrm{ES}+) \mathrm{m} / z(\%): 288 / 90 / 92 / 94$ $\left(\mathrm{M}+\mathrm{H}^{+}, 100\right)$. Anal. Calcd. for $\mathrm{C}_{13} \mathrm{H}_{12} \mathrm{NCl}_{3}: \mathrm{C}, 54.10 ; \mathrm{H}, 4.19 ; \mathrm{N}, 4.85$. Found: $\mathrm{C}, 54.33 ; \mathrm{H}$, $4.23 ; \mathrm{N}, 4.75$.

2,4,4-Trichloro-3-phenylcyclobutenone (15). A solution of $10.00 \mathrm{~g}(46.51 \mathrm{mmol})$ 2,2-dichloro-3-phenylcyclobutanone 10a in $100 \mathrm{ml}$ of chloroform containing $25.19 \mathrm{~g}(0.23$ mol, 5 equiv.) DMF- $\mathrm{HCl}$ and $25.19 \mathrm{~g} \mathrm{DMF}$ was heated until $35^{\circ} \mathrm{C}$ (protected from light) and a stream of $\mathrm{Cl}_{2}$-gas was bubbled through the solution under stirring. Cooling was applied when the reaction temperature exceeded $55^{\circ} \mathrm{C}$. Chlorination was continued until no temperature raise was observed when adding chlorine. After reaction (ca. 20-30 minutes) the mixture was poured in $100 \mathrm{ml}$ water and extracted with chloroform $(4 \times 50 \mathrm{ml})$. After washing of the extracts with sodium bisulfite, drying of the organic layer $\left(\mathrm{MgSO}_{4}\right)$ and evaporation of the solvent, trichlorocyclobutenone $\mathbf{1 5}$ was obtained which could be purified by flash chromatography over a short silica column. Flash chromatography (hexane/ $\mathrm{CHCl}_{3} 4: 1, \mathrm{R}_{\mathrm{f}}=$ 0.31); yield 79\%, mp: $125-126^{\circ} \mathrm{C} .{ }^{1} \mathrm{H}$ NMR $\left(300 \mathrm{MHz}, \mathrm{CDCl}_{3}\right) \delta$ 7.59-7.69 (3H, m), 8.16-8.20 
$(2 \mathrm{H}, \mathrm{m}) ;{ }^{13} \mathrm{C} \mathrm{NMR}\left(75 \mathrm{MHz}, \mathrm{CDCl}_{3}\right) \delta 87.9,125.4,127.6,2 \times 129.6,2 \times 130.2,134.4,169.7$,

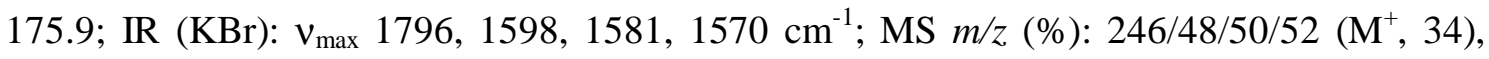
184/86/88 (15), 183/85/87 (100), 113 (33), 105 (32), 91 (16), 77 (51); Anal. Calcd. for $\mathrm{C}_{10} \mathrm{H}_{5} \mathrm{OCl}_{3}: \mathrm{C}, 48.53 ; \mathrm{H}, 2.04$. Found: $\mathrm{C}, 48.71 ; \mathrm{H}, 2.19$.

\section{Synthesis of 3-hydroxy-4-phenyl-3-cyclobutene-1,2-dione (1)}

3-Hydroxy-4-phenyl-3-cyclobutene-1,2-dione $\quad$ (1). ${ }^{28} \quad$ To $\quad$ hydrolyze trichlorocyclobutenone (15), an excess of $90 \%$ aqueous sulfuric acid (10 ml) was added to $2.00 \mathrm{~g}$ of trichlorocyclobutenone 15 and heated to $80^{\circ}-90^{\circ} \mathrm{C}$. After stirring for 15 hours, the acidic mixture was poured on crushed ice and extracted with chloroform $(3 \times 25 \mathrm{ml})$. The extracts were dried $\left(\mathrm{MgSO}_{4}\right)$ and the solvent evaporated, yielding almost pure 4phenylsemisquaric acid $\mathbf{1}$ as a crystalline compound. Purified by washing with cold diethyl ether; yield $91 \%, \mathrm{mp}: 210^{\circ} \mathrm{C}$ (decomp.)(Lit. ${ }^{28}$ : 206-208 ${ }^{\circ} \mathrm{C}$ ). Spectroscopic data were in accordance with literature data.

\section{Synthesis of $N$-(2,4,4-trialkoxy-2-cyclobutenylidene)amines 16}

$N$-(2,4,4-Trimethoxy-3-phenyl-2-cyclobutenylidene)isopropylamine (16a). To 0.32

g (0.98 mmol) of $N$-(2,2,4,4-tetrachloro-3-phenylcyclobutylidene)isopropylamine 13a was added dropwise $0.99 \mathrm{ml}$ (3.94 mmol, 4 equiv.) of $4 \mathrm{M} \mathrm{NaOMe}$ in methanol at $0^{\circ} \mathrm{C}$ (When working on big scale, e.g. $10 \mathrm{~g}$, it is appropriate to dissolve the tetrachlorinated imine 13a in a minimal amount of methanol, prior to the addition of sodium methoxide to control the reaction speed (exothermic reaction)). After reflux for 1 hour, the mixture was diluted with $25 \mathrm{ml}$ of water and extracted with dichloromethane $(4 \times 20 \mathrm{ml})$. The extracts were dried over a mixture of $\mathrm{K}_{2} \mathrm{CO}_{3}$ and $\mathrm{MgSO}_{4}$ and after filtration, the solvents were evaporated yielding cyclobutenimine $\mathbf{1 6 a}$, which could be purified by flash chromatography or by recrystallization 
in hexane/ethyl acetate (1:1). Flash chromatography (hexane/EtOAc 9:1, $\mathrm{R}_{\mathrm{f}}=0.19$ ); yield $88 \%, \mathrm{mp} 77-78^{\circ} \mathrm{C} .{ }^{1} \mathrm{H}$ NMR $\left(300 \mathrm{MHz}, \mathrm{CDCl}_{3}\right) \delta 1.19(6 \mathrm{H}, \mathrm{d}, J=6.3 \mathrm{~Hz}), 3.32(6 \mathrm{H}, \mathrm{s}), 3.97$ $(1 \mathrm{H}$, sept, $J=6.3 \mathrm{~Hz}), 4.32(3 \mathrm{H}, \mathrm{s}), 7.27-7.41(3 \mathrm{H}, \mathrm{m}), 7.62-7.66(2 \mathrm{H}, \mathrm{m}) ;{ }^{13} \mathrm{C} \mathrm{NMR}(75 \mathrm{MHz}$, $\left.\mathrm{CDCl}_{3}\right) \delta 2 \times 24.0,2 \times 52.5,52.6,59.3,112.2,2 \times 126.4,128.4,2 \times 128.7,131.0,134.1$, 156.0, 162.2; IR (KBr): $v_{\max } 1688,1612,1450 \mathrm{~cm}^{-1}$; MS m/z (\%): $275\left(\mathrm{M}^{+}, 11\right), 232(100)$, 186 (62), 144 (72), 128 (54), 115 (48), 89 (65), 77 (37); Anal. Calcd. for $\mathrm{C}_{16} \mathrm{H}_{21} \mathrm{NO}_{3}$ : C, 69.79; H, 7.69; N, 5.09. Found: C, 69.92; H, 7.82; N, 4.95.

$N$-(2,4,4-Triethoxy-3-phenyl-2-cyclobutenylidene)isopropylamine 16b. Flash chromatography (hexane/EtOAc 9:1, $\left.\mathrm{R}_{\mathrm{f}}=0.35\right)$; yield $66 \%, \mathrm{mp} 71-73^{\circ} \mathrm{C} .{ }^{1} \mathrm{H}$ NMR $(270 \mathrm{MHz}$, $\left.\mathrm{CDCl}_{3}\right) \delta 1.17(6 \mathrm{H}, \mathrm{d}, J=6.3 \mathrm{~Hz}), 1.20(6 \mathrm{H}, \mathrm{t}, J=6.9 \mathrm{~Hz}), 1.39(3 \mathrm{H}, \mathrm{t}, J=7.1 \mathrm{~Hz}), 3.45$ and $3.46(2 \times 2 \mathrm{H}, 2 \times \mathrm{q}, J=6.9 \mathrm{~Hz}), 4.05(1 \mathrm{H}, \mathrm{sept}, J=6.3 \mathrm{~Hz}), 4.70(2 \mathrm{H}, \mathrm{q}, J=7.1 \mathrm{~Hz}), 7.25-7.40$ $(3 \mathrm{H}, \mathrm{m}), 7.66-7.69(2 \mathrm{H}, \mathrm{m}) ;{ }^{13} \mathrm{C} \mathrm{NMR}\left(68 \mathrm{MHz}, \mathrm{CDCl}_{3}\right) \delta 2 \times 15.3,15.9,2 \times 24.0,52.4,2 \times$ $60.1,67.5,110.5,2 \times 126.3,128.3,2 \times 128.7,131.3,135.3,155.1,163.4 ; \mathrm{IR}(\mathrm{NaCl}): v_{\max }$ 1697, 1615, $1479 \mathrm{~cm}^{-1}$; MS m/z (\%): 317 (M+30), 274 (87), 246 (35), 218 (49), 200 (100), 190 (51), 172 (82), 145 (96), 117 (34); Anal. Calcd. for $\mathrm{C}_{19} \mathrm{H}_{27} \mathrm{NO}_{3}: \mathrm{C}, 71.89 ; \mathrm{H}, 8.57$; N, 4.41. Found: C, 71.74; H, 8.73; N, 4.30.

$N$-(2,4,4-Triisopropoxy-3-phenyl-2-cyclobutenylidene)isopropylamine 16c. Flash chromatography (hexane/EtOAc 95:5, $\left.\mathrm{R}_{\mathrm{f}}=0.25\right)$; yield $37 \% .{ }^{1} \mathrm{H} \mathrm{NMR}\left(270 \mathrm{MHz}, \mathrm{CDCl}_{3}\right) \delta$ $1.03(6 \mathrm{H}, \mathrm{d}, J=6.0 \mathrm{~Hz}), 1.17$ and $1.19(2 \times 6 \mathrm{H}, 2 \times \mathrm{d}, J=6.2 \mathrm{~Hz}), 1.38(6 \mathrm{H}, \mathrm{d}, J=6.2 \mathrm{~Hz}), 3.89$ $(2 \mathrm{H}$, sept, $J=6.2 \mathrm{~Hz}), 4.08(1 \mathrm{H}$, sept, $J=6.0 \mathrm{~Hz}), 5.57(1 \mathrm{H}$, sept, $J=6.2 \mathrm{~Hz}), 7.22-7.38(3 \mathrm{H}$, m), 7.67-7.71 (2H, m); ${ }^{13} \mathrm{C} \mathrm{NMR}\left(68 \mathrm{MHz}, \mathrm{CDCl}_{3}\right) \delta 2 \times 23.1,2 \times 24.0,4 \times 24.1,51.9,2 \times$ $67.3,73.8,110.5,2 \times 126.9,128.4,2 \times 128.6,132.1,137.1,154.9,164.9 ; \mathrm{IR}(\mathrm{NaCl}) v_{\max }$ 1696, $1612 \mathrm{~cm}^{-1}$; MS m/z (\%) $359\left(\mathrm{M}^{+}, 6\right), 316$ (17), 274 (19), 232 (50), 190 (76), 172 (63), 163 (68), 145 (95), 43 (100); Anal. Calcd. for $\mathrm{C}_{22} \mathrm{H}_{33} \mathrm{NO}_{3}$ : C, 73.50; H, 9.25; N, 3.90. Found: C, 73.69; H, 9.42; N, 3.75. 
$N$-(2,4,4-Trimethoxy-3-phenyl-2-cyclobutenylidene)cyclohexylamine 16d. Flash chromatography (hexane/EtOAc 9:1, $\left.\mathrm{R}_{\mathrm{f}}=0.30\right)$; yield $40 \%, \mathrm{mp} 72-73^{\circ} \mathrm{C} .{ }^{1} \mathrm{H}$ NMR $(270 \mathrm{MHz}$, $\left.\mathrm{CDCl}_{3}\right) \delta 1.26-1.81(10 \mathrm{H}, \mathrm{m}), 3.32(6 \mathrm{H}, \mathrm{s}), 3.56-3.67(1 \mathrm{H}, \mathrm{m}), 4.31(3 \mathrm{H}, \mathrm{s}), 7.26-7.40(3 \mathrm{H}$, m), 7.62-7.65 (2H, m); ${ }^{13} \mathrm{C} \mathrm{NMR}\left(68 \mathrm{MHz}, \mathrm{CDCl}_{3}\right) \delta 2 \times 24.5,25.8,2 \times 34.1,2 \times 52.5,59.3$, $30.7,112.2,2 \times 126.4,128.4,2 \times 128.7,131.0,134.2,156.1,162.3 ; \mathrm{IR}(\mathrm{NaCl}) v_{\max } 1700$, 1618, $1448 \mathrm{~cm}^{-1}$; MS m/z (\%) $315\left(\mathrm{M}^{+}, 15\right), 232$ (100), 218 (25), 55 (22), 45 (27), 43 (30); Anal. Calcd. for $\mathrm{C}_{19} \mathrm{H}_{25} \mathrm{NO}_{3}$ : C, 72.35; H, 7.99; N, 4.44. Found: C, 72.66; H, 8.15; N, 4.38.

$N$-(2,4,4-Trimethoxy-3-phenyl-2-cyclobutenylidene)-t-butylamine 16e. Flash chromatography (hexane/EtOAc 96:4, $\mathrm{R}_{\mathrm{f}}=0.19$ ); yield $36 \%, \mathrm{mp} 61-62^{\circ} \mathrm{C} .{ }^{1} \mathrm{H} \mathrm{NMR}$ $\left(270 \mathrm{MHz}, \mathrm{CDCl}_{3}\right) \delta 1.33(9 \mathrm{H}, \mathrm{s}), 3.29(6 \mathrm{H}, \mathrm{s}), 4.34(3 \mathrm{H}, \mathrm{s}), 7.27-7.39(3 \mathrm{H}, \mathrm{m}), 7.63-7.67$ $(2 \mathrm{H}, \mathrm{m}) ;{ }^{13} \mathrm{C} \mathrm{NMR}\left(68 \mathrm{MHz}, \mathrm{CDCl}_{3}\right) \delta 3 \times 30.2,2 \times 52.1,56.8,59.0,114.0,2 \times 126.1,128.2$, $2 \times 128.7,131.1,133.9,156.8,160.1 ; \mathrm{IR}(\mathrm{NaCl}) v_{\max } 1690,1619,1495,1450 \mathrm{~cm}^{-1} ; \mathrm{MS} \mathrm{m} / z$ (\%) 289 (M+, 6), 233 (19), 232 (100), $186(10), 142$ (11), 115 (10), 100 (10), 57 (21), 41 (19); Anal. Calcd. for $\mathrm{C}_{17} \mathrm{H}_{23} \mathrm{NO}_{3}$ : C, 70.56; H, 8.01; N, 4.84. Found: C, 70.29; H, 8.16; N, 4.74.

$N$-(3-(4-Chlorophenyl)-2,4,4-trimethoxy-2-cyclobutenylidene)isopropylamine 16 .

Flash chromatography (hexane/EtOAc 9:1, $\mathrm{R}_{\mathrm{f}}=0.41$ ); yield $79 \%, \mathrm{mp} 108-110^{\circ} \mathrm{C} .{ }^{1} \mathrm{H} \mathrm{NMR}$ $\left(300 \mathrm{MHz}, \mathrm{CDCl}_{3}\right) \delta 1.19(6 \mathrm{H}, \mathrm{d}, J=6.3 \mathrm{~Hz}), 3.31(6 \mathrm{H}, \mathrm{s}), 3.95(1 \mathrm{H}, \mathrm{sept}, J=6.3 \mathrm{~Hz}), 4.32$ $(3 \mathrm{H}, \mathrm{s}), 7.33(2 \mathrm{H}, \mathrm{d}, J=8.6 \mathrm{~Hz}), 7.57(2 \mathrm{H}, \mathrm{d}, J=8.6 \mathrm{~Hz}) ;{ }^{13} \mathrm{C} \mathrm{NMR}\left(75 \mathrm{MHz}, \mathrm{CDCl}_{3}\right): \delta 2 \times$ $24.1,2 \times 52.6,52.8,59.5,112.1,2 \times 127.7,2 \times 129.1,129.4,132.8,134.2,156.3,162.0 ;$ IR (KBr): $v_{\max } 1697,1620,1372 \mathrm{~cm}^{-1} . \mathrm{MS}(\mathrm{ES}+) \mathrm{m} / z(\%): 310\left(\mathrm{M}+\mathrm{H}^{+}, 100\right)$. Anal. Calcd. for $\mathrm{C}_{16} \mathrm{H}_{20} \mathrm{NO}_{3} \mathrm{Cl}: \mathrm{C}, 62.03 ; \mathrm{H}, 6.51 ; \mathrm{N}, 4.52$. Found: C, 61.89; H, 6.70; N, 4.35.

\section{Synthesis of semisquarates 17 and semisquaramide 18}

3-Methoxy-4-phenyl-3-cyclobutene-1,2-dione $\quad(17 a) .{ }^{29 a} \quad N-(2,4,4-T r i m e t h o x y-3-$ phenyl-2-cyclobutenylidene)isopropylamine 16a (3.92 g, $14.26 \mathrm{mmol}$ ) was dissolved in $50 \mathrm{ml}$ 
of dichloromethane and $50 \mathrm{ml}$ of aqueous $2 \mathrm{M} \mathrm{HCl}$. The biphasic mixture was refluxed for 2 hours and subsequently, the organic layer was separated after cooling. The aqueous phase was extracted with dichloromethane $(2 \times 50 \mathrm{ml})$ and the combined extracts were dried over $\mathrm{MgSO}_{4}$. After filtration and evaporation of the solvent in vacuo, 3-methoxy-4phenylcyclobuten-1,2-dione 17a was obtained as a yellow solid which could be purified by recrystallization. Recrystallization (diethyl ether/hexane 1:1); yield $72 \%, \mathrm{mp} 152-153^{\circ} \mathrm{C}$ $\left(\right.$ Lit. $\left.^{29 \mathrm{a}}: 150-151^{\circ} \mathrm{C}\right)$. Spectral data were in accordance with literature data. ${ }^{29 \mathrm{a}}$

3-Ethoxy-4-phenyl-3-cyclobutene-1,2-dione $\quad \mathbf{1 7 b}^{29 \mathrm{~b}}$ Flash chromatography (hexane/EtOAc 4:1, $\mathrm{R}_{\mathrm{f}}=0.29$ ); yield $73 \%$, mp $133-134^{\circ} \mathrm{C}$ (no lit. data). Spectral data were in accordance with literature data. ${ }^{29 b}$

3-Isopropoxy-4-phenyl-3-cyclobutene-1,2-dione 17 . $^{29 a}$ Flash chromatography (hexane/EtOAc 4:1, $\mathrm{R}_{\mathrm{f}}=0.31$ ); yield $60 \%$, mp 106-107 ${ }^{\circ} \mathrm{C}$ (no lit. data). Spectral data were in accordance with literature data. ${ }^{29 a}$

\section{4-(4-Chlorophenyl)-3-methoxy-3-cyclobuten-1,2-dione $\quad$ 17d. $^{29 \mathrm{c}} \quad$ Flash} chromatography (hexane/EtOAc 4:1, $\mathrm{R}_{\mathrm{f}}=0.20$ ); yield $78 \%, \mathrm{mp} 126^{\circ} \mathrm{C}$ (no lit. data). Spectral data were in accordance with literature data. ${ }^{29 c}$

3-Isopropylamino-4-phenyl-3-cyclobutene-1,2-dione 18. Cyclobutenedione 17a $(0.50 \mathrm{~g}, 2.66 \mathrm{mmol})$ was dissolved in $5 \mathrm{ml}$ of isopropylamine and stirred for 10 minutes at room temperature. The complete reaction mixture was evaporated in vacuo leaving pure 3isopropylaminocyclobutenedione 18 in quantitative yield. $\mathrm{Mp} 189^{\circ} \mathrm{C} .{ }^{1} \mathrm{H}$ NMR $(300 \mathrm{MHz}$, $\left.\mathrm{CDCl}_{3}\right) \delta 1.40(6 \mathrm{H}, \mathrm{d}, J=6.6 \mathrm{~Hz}), 4.68(1 \mathrm{H}$, sept $\times \mathrm{d}, J=6.6 \mathrm{~Hz}, 8.6 \mathrm{~Hz}), 6.33(1 \mathrm{H}, \mathrm{d}(\mathrm{b}), J=$ 8.6Hz), 7.43-7.51 (3H, m), 7.81-7.85 (2H, m). $\left.{ }^{13} \mathrm{C} \mathrm{NMR} \mathrm{(75MHz,} \mathrm{CDCl}_{3}\right) \delta 2 \times 24.0,48.1,2$ $\times 126.1,2 \times 129.1,129.3,130.8,162.9,179.3,189.6,191.8 . \mathrm{IR}(\mathrm{KBr}): v_{\max } 3436,3150,1771$, 1720, 1610, $1582 \mathrm{~cm}^{-1}$. MS (ES+) m/z (\%): $254\left(\mathrm{M}+\mathrm{K}^{+}, 21\right), 238\left(\mathrm{M}+\mathrm{Na}^{+}, 7\right), 216\left(\mathrm{M}+\mathrm{H}^{+}\right.$, 
100). Anal. Calcd. for $\mathrm{C}_{13} \mathrm{H}_{13} \mathrm{NO}_{2}: \mathrm{C}, 72.54 ; \mathrm{H}, 6.09 ; \mathrm{N}, 6.51$. Found: $\mathrm{C}, 72.36 ; \mathrm{H}, 6.18 ; \mathrm{N}$, 6.36.

\section{Synthesis of $N$-(cyclobutylidene)amines 20}

$N$-(Cyclobutylidene)isopropylamine $20 \mathrm{a}^{30}$ To a solution of $2.50 \mathrm{~g}(35.71 \mathrm{mmol})$ cyclobutanone 19 in $50 \mathrm{ml}$ of dry diethyl ether was added $8.26 \mathrm{~g}$ (0.14 mmol, 4 equiv.) isopropylamine. The solution was cooled to $-20^{\circ} \mathrm{C}$ with the use of a mixture of $\mathrm{NaCl} /$ ice. To the cooled solution was added dropwise $4.07 \mathrm{~g}$ (21.43 mmol, 0.6 equiv.) titanium(IV) chloride in $10 \mathrm{ml}$ of dry petroleum ether over a period of 15 minutes. After stirring the reaction mixture for 3 hours during which the temperature raised to $0^{\circ} \mathrm{C}$, the reaction mixture was poured in $100 \mathrm{ml}$ aq. $0.5 \mathrm{M} \mathrm{NaOH}$ and was rapidly extracted with $4 \times 50 \mathrm{ml}$ of diethyl ether. After each extraction, the solvent was collected in an erlenmeyer containing a mixture of $\mathrm{MgSO}_{4}$ and $\mathrm{K}_{2} \mathrm{CO}_{3}$. After extraction the combined organic phases were filtered and dried again over $\mathrm{MgSO}_{4} / \mathrm{K}_{2} \mathrm{CO}_{3}$. The extraction procedure was performed fast, to minimize the time that the formed imine 20a was in contact with water. After careful evaporation of the solvent at low vacuum $(30 \mathrm{mmHg})$ at ca. $10^{\circ} \mathrm{C}$, a pale yellow oil was obtained which was used as such, without further purification. In the case of the $n$-propyl and isobutyl derivatives $\mathbf{2 0 b}$ and 20c, no full spectroscopic characterization was possible due to the lability of the compounds. Other derivatives $(\mathbf{2 0 a}, \mathbf{2 0 d}$ and $20 \mathrm{e})$ were more stable and could even be distilled (20d and 20e) prior to further transformations. ${ }^{1} \mathrm{H}$ NMR $\left(300 \mathrm{MHz}, \mathrm{CDCl}_{3}\right) \delta 1.11(6 \mathrm{H}, \mathrm{d}, J=6.3 \mathrm{~Hz})$, 1.89-2.04 (2H, m), 2.83-2.97 (4H, m), $3.42(1 \mathrm{H}$, sept, $J=6.3 \mathrm{~Hz}) ;{ }^{13} \mathrm{C} \mathrm{NMR}\left(75 \mathrm{MHz}, \mathrm{CDCl}_{3}\right)$ $\delta 13.0,23.3,23.4,33.9,37.6,51.1,168.7$; IR $(\mathrm{NaCl}): v_{\max } 1705 \mathrm{~cm}^{-1} ; \mathrm{MS} m / z(\%): 111\left(\mathrm{M}^{+}\right.$, 2), 84 (2), 83 (17), 43 (29), 41 (100).

$\mathrm{N}$-Cyclobutylidene-2-butanamine 20d. Distillation $\left(50-53^{\circ} \mathrm{C}, 10 \mathrm{mmHg}\right)$; yield $73 \%$.

${ }^{1} \mathrm{H}$ NMR $\left(300 \mathrm{MHz}, \mathrm{CDCl}_{3}\right) \delta 0.82(3 \mathrm{H}, \mathrm{t}, J=7.4 \mathrm{~Hz}), 1.08(3 \mathrm{H}, \mathrm{d}, J=6.4 \mathrm{~Hz}), 1.37-1.55(2 \mathrm{H}$, 
m), $1.95(2 \mathrm{H}$, quint, $J=8.1 \mathrm{~Hz}), 2.82-2.97(4 \mathrm{H}, \mathrm{m}), 3.12(1 \mathrm{H}$, sext, $J=6.4 \mathrm{~Hz}) ;{ }^{13} \mathrm{C} \mathrm{NMR}$ $\left(\mathrm{CDCl}_{3}\right) \delta 10.7,10.9,21.2,30.4,34.3,37.6,57.4,168.5 ; \mathrm{IR}(\mathrm{NaCl}): v_{\max } 1714 \mathrm{~cm}^{-1} ; \mathrm{MS} \mathrm{m} / z$ (\%): $125\left(\mathrm{M}^{+}, 6\right), 97(85), 69(21), 57$ (75), 41 (100).

$N$-Cyclobutylidene-2-methyl-2-propanamine 20e. Distillation $\left(64-65^{\circ} \mathrm{C}, 35 \mathrm{mmHg}\right)$; yield $80 \% .{ }^{1} \mathrm{H}$ NMR $\left(300 \mathrm{MHz}, \mathrm{CDCl}_{3}\right) \delta 1.22(9 \mathrm{H}, \mathrm{s}), 1.93(2 \mathrm{H}$, quint, $J=8.2 \mathrm{~Hz}), 2.88-2.69$ $(2 \mathrm{H}, \mathrm{m}), 3.00-3.08(2 \mathrm{H}, \mathrm{m}) ;{ }^{13} \mathrm{C} \mathrm{NMR}\left(75 \mathrm{MHz}, \mathrm{CDCl}_{3}\right) \delta 13.5,3 \times 27.8,38.5,39.7,55.8$, 167.7; IR (NaCl): $v_{\max } 1712 \mathrm{~cm}^{-1} ; \mathrm{MS} \mathrm{m} / z(\%): 125\left(\mathrm{M}^{+}, 5\right), 110(6), 97$ (23), 57 (100).

\section{Chlorination of imines 20}

$N$-(2,2,4,4-Tetrachlorocyclobutylidene)isopropylamine 21a. To a solution of $1.00 \mathrm{~g}$ (9.01 mmol) $\mathrm{N}$-(cyclobutylidene)isopropylamine $20 \mathrm{a}$ in $50 \mathrm{ml}$ of dry $\mathrm{CCl}_{4}$ was added $5.29 \mathrm{~g}$ (45.05 mmol, 5 equiv.) of NCS in one portion. The flask was immediately immersed in a preheated oil bath and the mixture was refluxed for 30 minutes. Attention must be drawn to the fact that when this procedure is applied on larger scales, the exothermicity of the reaction can cause a rapid refluxing of the $\mathrm{CCl}_{4}$, so cooling can be neccessary in some cases. After reaction, the mixture was cooled in an ice bath and subsequently filtered. The filtrate was cooled again at $-20^{\circ} \mathrm{C}$ for 2 hours and some more succinimide was filtered. After filtration, the filtrate was dried over $\mathrm{MgSO}_{4}$, filtered and the $\mathrm{CCl}_{4}$ was evaporated in vacuo at ca. $10^{\circ} \mathrm{C}$. Yellow crystals were obtained which could be recrystallized. Also in this case, derivatives 21b and 21c were too labile and could not be purified. Recrystallization (hexane/CHCl 3 9:1); yield $88 \%$, mp $75-77^{\circ} \mathrm{C} .{ }^{1} \mathrm{H}$ NMR $\left(300 \mathrm{MHz}, \mathrm{CDCl}_{3}\right) \delta 1.32(6 \mathrm{H}, \mathrm{d}, J=5.9 \mathrm{~Hz}), 3.73(2 \mathrm{H}, \mathrm{s})$, $4.53(1 \mathrm{H}$, sept, $J=5.9 \mathrm{~Hz}) ;{ }^{13} \mathrm{C} \mathrm{NMR}\left(75 \mathrm{MHz}, \mathrm{CDCl}_{3}\right) \delta 2 \times 22.6,51.7,64.8,2 \times 74.0,159.2$;

IR (KBr): $v_{\max } 1690 \quad \mathrm{~cm}^{-1}$; MS m/z (\%): no $\mathrm{M}^{+}, 151 / 53 / 55$ (18), 109/11/13 (25), 43 (100); Anal. Calcd. for $\mathrm{C}_{7} \mathrm{H}_{9} \mathrm{NCl}_{4}$ : C, 33.77; H, 3.64; N, 5.63. Found: C, 33.65; H, 3.55; N, 5.49. 
$N$-(2,2,4,4-Tetrachlorocyclobutylidene)-2-butanamine 21d. No purification; yield 94\%. ${ }^{1} \mathrm{H}$ NMR (300MHz, $\left.\mathrm{CDCl}_{3}\right) \delta 0.93(3 \mathrm{H}, \mathrm{t}, J=7.4 \mathrm{~Hz}), 1.30(3 \mathrm{H}, \mathrm{d}, J=6.3 \mathrm{~Hz}), 1.65-1.75$ $(2 \mathrm{H}, \mathrm{m}), 3.74(2 \mathrm{H}, \mathrm{s}), 4.25(1 \mathrm{H}, \mathrm{sext}, J=6.3 \mathrm{~Hz}) ;{ }^{13} \mathrm{C} \mathrm{NMR}\left(75 \mathrm{MHz}, \mathrm{CDCl}_{3}\right) \delta 11.0,20.6$, 30.5, 57.4, 64.9, $2 \times 74.2,159.8 ;$ IR $(\mathrm{NaCl}): v_{\max } 1709,1451 \mathrm{~cm}^{-1} ; \operatorname{MS~m} / z(\%)$ : 261/63/65/67/69 (M+, 1), 226/28/30/32 (M+Cl, 3), 165 (39), 57 (100), 41 (31).

$N$-(2,2,4,4-Tetrachlorocyclobutylidene)-2-methyl-2-propanamine 21e. Recrystallization (pentane/Et $2 \mathrm{O}$ ); yield $80 \%, \mathrm{mp} 55^{\circ} \mathrm{C} .{ }^{1} \mathrm{H}$ NMR $\left(300 \mathrm{MHz}, \mathrm{CDCl}_{3}\right) \delta 1.51$ $(9 \mathrm{H}, \mathrm{s}), 3.75(2 \mathrm{H}, \mathrm{s}) ;{ }^{13} \mathrm{C} \mathrm{NMR}\left(75 \mathrm{MHz}, \mathrm{CDCl}_{3}\right) \delta 3 \times 30.5,59.6,65.1,2 \times 77.4,153.6$; IR (KBr): $v_{\max } 1710 \mathrm{~cm}^{-1} ; \mathrm{MS} \mathrm{m/z}(\%): 261 / 63 / 65 / 67 / 69\left(\mathrm{M}^{+}, 1\right), 246 / 48 / 50 / 52 / 54\left(\mathrm{M}^{+}-\mathrm{CH}_{3}, 5\right)$, $57(100)$.

\section{Synthesis of aminocyclobutenones 29}

3-Isopropylamino-4,4-dimethoxy-2-cyclobuten-1-one 29a. To 1.54 g $(6.18 \mathrm{mmol})$ $N$-(2,2,4,4-tetrachlorocyclobutylidene)isopropylamine 21a (without solvent) was added dropwise (slowly, exothermic reaction!) $15.46 \mathrm{ml}$ (61.85 mmol, 10 equiv.) of $4 \mathrm{M} \mathrm{MeOH}$ in methanol at $0^{\circ} \mathrm{C}$. After reaction for 15 minutes at $0^{\circ} \mathrm{C}$, the mixture was refluxed for 1 hour. Standard workup by pouring the reaction mixture in $50 \mathrm{ml}$ water and extraction with dichloromethane, yielded dimethoxycyclobutenone 29a after drying $\left(\mathrm{MgSO}_{4}\right)$ and evaporation of the solvents in vacuo. Recrystallization or flash chromatography could be used as a purification step. Flash chromatography (hexane/EtOAc $1: 1, \mathrm{R}_{\mathrm{f}}=0.11$ ); yield $54 \%, \mathrm{mp} 91^{\circ} \mathrm{C}$.

${ }^{1} \mathrm{H} \mathrm{NMR}\left(300 \mathrm{MHz}, \mathrm{CDCl}_{3}\right) \delta 1.28(6 \mathrm{H}, \mathrm{d}, J=6.6 \mathrm{~Hz}), 3.51(6 \mathrm{H}, \mathrm{s}), 3.54(1 \mathrm{H}, \mathrm{sept}, J=6.6 \mathrm{~Hz})$, $5.20(1 \mathrm{H}, \mathrm{s}), 5.72\left(1 \mathrm{H}, \mathrm{s}(\mathrm{b}) ;{ }^{13} \mathrm{C} \mathrm{NMR}\left(75 \mathrm{MHz}, \mathrm{CDCl}_{3}\right) \delta 2 \times 21.5,47.5,2 \times 52.6,106.9\right.$, 112.9, 172.6, 188.7; IR (KBr): $v_{\max } 3248,1744 \mathrm{~cm}^{-1}$; MS m/z (\%): $185\left(\mathrm{M}^{+}, 1\right), 170(100)$, 154 (37), 128 (17), 100 (89); Anal. Calcd. for $\mathrm{C}_{9} \mathrm{H}_{15} \mathrm{NO}_{3}$ : C, 58.36; H, 8.16; N, 7.56. Found: C, 58.21; H, 8.10; N, 7.66. 
3-(Sec-butylamino)-4,4-dimethoxy-2-cyclobuten-1-one 29d. Flash chromatography (hexane/EtOAc 1:1, $\left.\mathrm{R}_{\mathrm{f}}=0.14\right)$; yield $61 \% .{ }^{1} \mathrm{H}$ NMR $\left(300 \mathrm{MHz}, \mathrm{CDCl}_{3}\right) \delta 0.97(3 \mathrm{H}, \mathrm{t}, J=$ 7.4Hz), $1.27(3 \mathrm{H}, \mathrm{d}, J=6.6 \mathrm{~Hz}), 1.61(2 \mathrm{H}, \mathrm{dq}, J=7.4 \mathrm{~Hz}, 6.6 \mathrm{~Hz}), 3.26-3.41(1 \mathrm{H}, \mathrm{m}), 3.51(3 \mathrm{H}$, s), $3.52(3 \mathrm{H}, \mathrm{s}), 5.19(1 \mathrm{H}, \mathrm{s}), 5.80(1 \mathrm{H}, \mathrm{s}(\mathrm{b})) ;{ }^{13} \mathrm{C} \mathrm{NMR}\left(75 \mathrm{MHz}, \mathrm{CDCl}_{3}\right) \delta 10.5,19.7,28.9,2$ $\times 52.8,53.9,106.9,113.2,173,188.1 ; \mathrm{IR}(\mathrm{NaCl}): v_{\max } 3237,1740,1590 \mathrm{~cm}^{-1} ; \mathrm{MS} \mathrm{m} / \mathrm{z}(\%):$ $199\left(\mathrm{M}^{+}, 1\right), 184\left(\mathrm{M}^{+}-\mathrm{CH}_{3}, 100\right), 100$ (95); Anal. Calcd. for $\mathrm{C}_{10} \mathrm{H}_{17} \mathrm{NO}_{3}: \mathrm{C}, 60.28 ; \mathrm{H}, 8.60 ; \mathrm{N}$, 7.03. Found: C, 60.43; H, 8.79; N, 6.86.

3-(Tert-butylamino)-4,4-dimethoxy-2-cyclobuten-1-one 29e. Recrystallization (diethyl ether); yield $64 \%, \mathrm{mp} 134-136^{\circ} \mathrm{C} .{ }^{1} \mathrm{H}$ NMR $\left(300 \mathrm{MHz}, \mathrm{CDCl}_{3}\right) \delta 1.38(9 \mathrm{H}, \mathrm{s}), 3.50$ $(6 \mathrm{H}, \mathrm{s}), 5.26(1 \mathrm{H}, \mathrm{s}), 6.04\left(1 \mathrm{H}, \mathrm{s}(\mathrm{b}) .{ }^{13} \mathrm{C} \mathrm{NMR}\left(75 \mathrm{MHz}, \mathrm{CDCl}_{3}\right) \delta 3 \times 28.6,2 \times 53.5,53.6\right.$, 109.6, 114.7, 171.3, 188.3; IR (KBr): $v_{\max } 3197,1733,1587 \mathrm{~cm}^{-1}$; MS m/z (\%): no $\mathrm{M}^{+}, 184$ $\left(\mathrm{M}^{+}-\mathrm{CH}_{3}, 14\right), 168$ (23), 128 (100). 100 (66); Anal. Calcd. for $\mathrm{C}_{10} \mathrm{H}_{17} \mathrm{NO}_{3}$ : C, 60.28; H, 8.60; N, 7.03. Found: C, 60.43; H, 8.77; N, 6.86.

\section{Synthesis of semisquaramides 30}

3-Isopropylamino-3-cyclobutene-1,2-dione 30a. To hydrolyze 3-isopropylamino4,4-dimethoxy-2-cyclobuten-1-one 29a, $0.50 \mathrm{~g}$ (2.70 mmol) was dissolved in $25 \mathrm{ml}$ of dichloromethane and an excess of aq. $2 \mathrm{M} \mathrm{HCl}$ was added $(10 \mathrm{ml})$. The biphasic system was refluxed for 1 hour, cooled down and extracted with dichloromethane $(3 \times 20 \mathrm{ml})$. After drying of the organic phase over $\mathrm{MgSO}_{4}$ and evaporation of the solvent, cyclobutenedione 30a was obtained as white-yellow crystals, which were purified by flash chromatography. Flash chromatography (hexane/EtOAc $1: 1, \mathrm{R}_{\mathrm{f}}=0.19$ ); yield $75 \%$, mp $91{ }^{\circ} \mathrm{C} .{ }^{1} \mathrm{H} \mathrm{NMR}$ $\left(300 \mathrm{MHz}, \mathrm{CDCl}_{3}\right) \delta 1.35(6 \mathrm{H}, \mathrm{d}, J=6.6 \mathrm{~Hz}), 3.68(1 \mathrm{H}, \mathrm{sept}, J=6.6 \mathrm{~Hz}), 6.51(1 \mathrm{H}, \mathrm{s}(\mathrm{b})), 8.00$ $(1 \mathrm{H}, \mathrm{s}) ;{ }^{13} \mathrm{C} \mathrm{NMR}\left(75 \mathrm{MHz}, \mathrm{CDCl}_{3}\right) \delta 2 \times 22.0,49.0,151.6,183.6,190.8,195.9 ; \mathrm{IR}(\mathrm{KBr}):$ $v_{\max } 3223,1776,1740 \mathrm{~cm}^{-1}$; MS m/z (\%): no $\mathrm{M}^{+}, 111\left(\mathrm{M}^{+}-\mathrm{CO}, 75\right), 96(3), 83(27), 68(100)$, 
43 (23); Anal. Calcd. for $\mathrm{C}_{7} \mathrm{H}_{9} \mathrm{NO}_{2}$ : C, 60.42; H, 6.52; N, 10.07. Found: $\mathrm{C}, 60.25 ; \mathrm{H}, 6.68 ; \mathrm{N}$, 10.20 .

3-Propylamino-3-cyclobutene-1,2-dione 30b. Flash chromatography (hexane/EtOAc $\left.1: 1, \mathrm{R}_{\mathrm{f}}=0.12\right)$; yield $25 \%, \mathrm{mp} 81^{\circ} \mathrm{C} .{ }^{1} \mathrm{H}$ NMR $\left(300 \mathrm{MHz}, \mathrm{CDCl}_{3}\right) \delta 1.01(3 \mathrm{H}, \mathrm{t}, J=7.4 \mathrm{~Hz})$, 1.68-1.80 (2H, m), $3.34(2 \mathrm{H}, \mathrm{q}, J=6.7 \mathrm{~Hz}), 7.47(1 \mathrm{H}, \mathrm{s}(\mathrm{b})), 8.01(1 \mathrm{H}, \mathrm{s}) ;{ }^{13} \mathrm{C} \mathrm{NMR}\left(\mathrm{CDCl}_{3}\right): \delta$ 11.3, 22.3, 48.2, 151.5, 185.4, 191.1, 195.3; IR (KBr): $v_{\max } 3185,3099,1781,1743,1733$, $1608 \mathrm{~cm}^{-1}$; MS m/z (\%): no $\mathrm{M}^{+}, 111\left(\mathrm{M}^{+}-\mathrm{CO}, 22\right), 82$ (40), 68 (77), 54 (100); Anal. Calcd. for $\mathrm{C}_{7} \mathrm{H}_{9} \mathrm{NO}_{2}: \mathrm{C}, 60.42 ; \mathrm{H}, 6.52 ; \mathrm{N}, 10.07$. Found: C, 60.54; H, 6.48; N, 9.97.

3-Isobutylamino-3-cyclobutene-1,2-dione 30c. Flash chromatography (hexane/EtOAc 1:1, $\left.\mathrm{R}_{\mathrm{f}}=0.17\right)$; yield $34 \%, \mathrm{mp} 82-83^{\circ} \mathrm{C} .{ }^{1} \mathrm{H}$ NMR $\left(300 \mathrm{MHz}, \mathrm{CDCl}_{3}\right) \delta 1.00$ $(6 \mathrm{H}, \mathrm{d}, J=6.9 \mathrm{~Hz}), 1.85-2.05(1 \mathrm{H}, \mathrm{m}), 3.18(2 \mathrm{H}, \mathrm{t}, J=6.5 \mathrm{~Hz}), 6.95\left(1 \mathrm{H}, \mathrm{s}(\mathrm{b}), 7.98(1 \mathrm{H}, \mathrm{s}) ;{ }^{13} \mathrm{C}\right.$ NMR $\left(75 \mathrm{MHz}, \mathrm{CDCl}_{3}\right) \delta 2 \times 20.0,28.7,54.1,151.9,185.6,190.5,195.4 ; \mathrm{IR}(\mathrm{KBr}): v_{\max }$ 3190, 1795, 1776, 1739, 1730, $1605 \mathrm{~cm}^{-1}$; MS m/z (\%): no $\mathrm{M}^{+}, 125\left(\mathrm{M}^{+}-\mathrm{CO}, 5\right), 84$ (23), 68 (41), 44 (100); Anal. Calcd. for $\mathrm{C}_{8} \mathrm{H}_{11} \mathrm{NO}_{2}$ : C, 62.73; H, 7.24; N, 9.14. Found: C, 62.66; H, $7.40 ; \mathrm{N}, 9.01$.

3-(Sec-butylamino)-3-cyclobuten-1,2-dione 30d. Flash chromatography (hexane/EtOAc 1:1, $\left.\mathrm{R}_{\mathrm{f}}=0.19\right)$; yield $81 \%, \mathrm{mp} 65-66^{\circ} \mathrm{C} .{ }^{1} \mathrm{H}$ NMR $\left(300 \mathrm{MHz}, \mathrm{CDCl}_{3}\right) \delta 0.98$ $(3 \mathrm{H}, \mathrm{t}, J=7.4 \mathrm{~Hz}), 1.33(3 \mathrm{H}, \mathrm{d}, J=6.6 \mathrm{~Hz}), 1.56-1.73(2 \mathrm{H}, \mathrm{m}), 3.36-3.50(1 \mathrm{H}, \mathrm{m}), 7.07(1 \mathrm{H}$, s(b)), $7.99(1 \mathrm{H}, \mathrm{s}) ;{ }^{13} \mathrm{C}$ NMR $\left(75 \mathrm{MHz}, \mathrm{CDCl}_{3}\right) \delta 10.5,19.7,29.2,55.1,151.3,184.2,191.2$, 195.6; IR (KBr): $v_{\max } 3205,1778,1746,1615 \mathrm{~cm}^{-1}$; MS m/z (\%): $153\left(\mathrm{M}^{+}, 1\right), 125\left(\mathrm{M}^{+}-\mathrm{CO}\right.$, 10), 110 (12), 84 (19), 68 (100); Anal. Calcd. for $\mathrm{C}_{8} \mathrm{H}_{11} \mathrm{NO}_{2}$ : C, 62.73; H, 7.24; N, 9.14. Found: C, 62.50; H, 7.43; N, 8.98.

3-(Tert-butylamino)-3-cyclobutene-1,2-dione 30e. ${ }^{31}$ Recrystallization (diethyl ether); yield $80 \%$, mp $162-163^{\circ} \mathrm{C}$ (Lit. ${ }^{31}: 165-167^{\circ} \mathrm{C}$ ). Spectral data were in accordance with literature data. ${ }^{31}$ 
III. X-ray crystallographic data of compound 29a

1. General view (ORTEP)

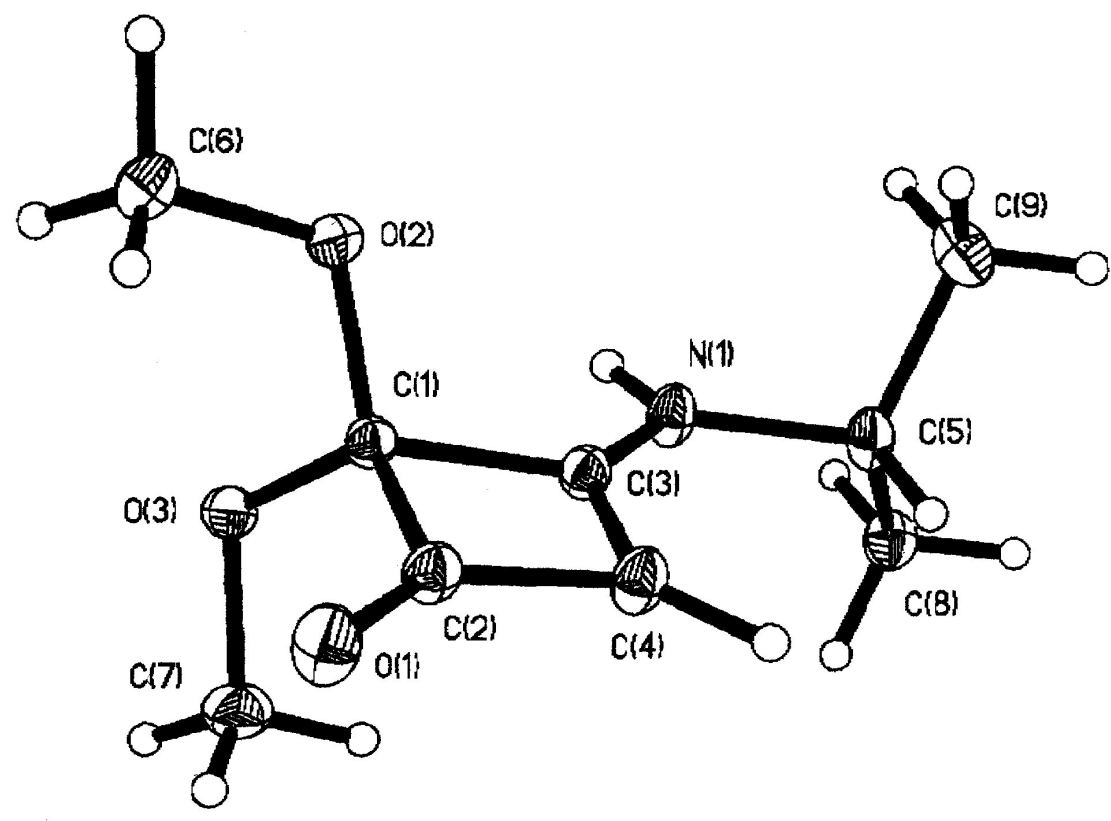

\section{Hydrogen bonding}

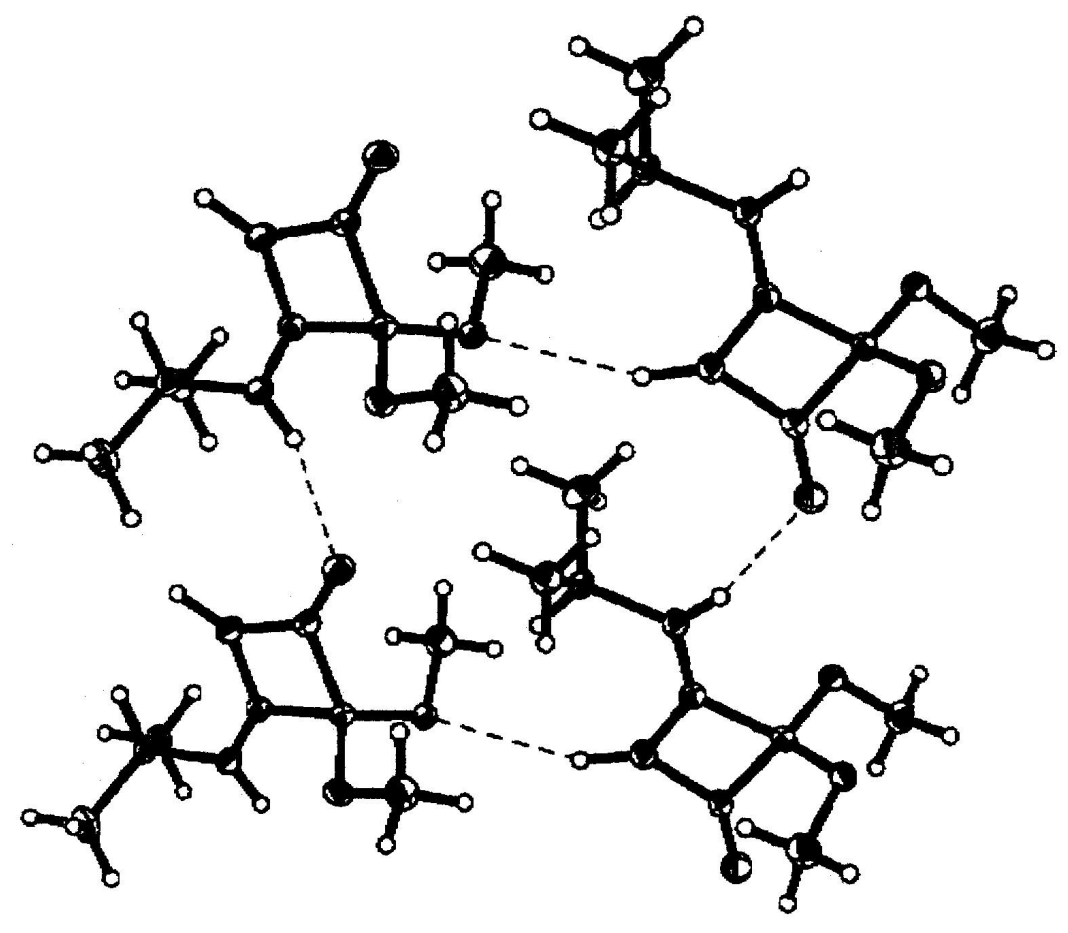


3. Bond lenghts $[\AA]$ and angles $\left[{ }^{\circ}\right]$

\begin{tabular}{|c|c|c|c|}
\hline $\mathrm{O}(3)-\mathrm{C}(1)$ & $1.4040(12)$ & $\mathrm{O}(1)-\mathrm{C}(2)-\mathrm{C}(4)$ & $137.40(10)$ \\
\hline $\mathrm{O}(3)-\mathrm{C}(7)$ & $1.4366(13)$ & $\mathrm{O}(1)-\mathrm{C}(2)-\mathrm{C}(1)$ & $132.18(9)$ \\
\hline $\mathrm{O}(1)-\mathrm{C}(2)$ & $1.2339(12)$ & $C(4)-C(2)-C(1)$ & $90.42(8)$ \\
\hline $\mathrm{O}(2)-\mathrm{C}(1)$ & $1.3935(11)$ & $\mathrm{N}(1)-\mathrm{C}(3)-\mathrm{C}(4)$ & $135.87(10)$ \\
\hline $\mathrm{O}(2)-\mathrm{C}(6)$ & $1.4339(13)$ & $N(1)-C(3)-C(1)$ & $130.06(9)$ \\
\hline$N(1)-C(3)$ & $1.3171(13)$ & $C(4)-C(3)-C(1)$ & $94.05(8)$ \\
\hline$N(1)-C(5)$ & $1.4777(13)$ & $C(3)-C(4)-C(2)$ & $92.98(8)$ \\
\hline $\mathrm{N}(1)-\mathrm{H}(1)$ & 0.8728 & $\mathrm{C}(3)-\mathrm{C}(4)-\mathrm{H}(4)$ & 133.5 \\
\hline$C(1)-C(3)$ & $1.5276(14)$ & $\mathrm{C}(2)-\mathrm{C}(4)-\mathrm{H}(4)$ & 133.5 \\
\hline$C(1)-C(2)$ & $1.5764(13)$ & $N(1)-C(5)-C(8)$ & $110.31(8)$ \\
\hline$C(2)-C(4)$ & $1.4318(14)$ & $N(1)-C(5)-C(9)$ & $109.15(9)$ \\
\hline$C(3)-C(4)$ & $1.3907(13)$ & $C(8)-C(5)-C(9)$ & $112.54(9)$ \\
\hline $\mathrm{C}(4)-\mathrm{H}(4)$ & 0.9765 & $\mathrm{~N}(1)-\mathrm{C}(5)-\mathrm{H}(5)$ & 108.2 \\
\hline$C(5)-C(8)$ & $1.5226(15)$ & $\mathrm{C}(8)-\mathrm{C}(5)-\mathrm{H}(5)$ & 108.2 \\
\hline$C(5)-C(9)$ & $1.5235(15)$ & $\mathrm{C}(9)-\mathrm{C}(5)-\mathrm{H}(5)$ & 108.2 \\
\hline $\mathrm{C}(5)-\mathrm{H}(5)$ & 1.0000 & $\mathrm{O}(2)-\mathrm{C}(6)-\mathrm{H}(6 \mathrm{~A})$ & 109.5 \\
\hline $\mathrm{C}(6)-\mathrm{H}(6 \mathrm{~A})$ & 0.9800 & $\mathrm{O}(2)-\mathrm{C}(6)-\mathrm{H}(6 \mathrm{~B})$ & 109.5 \\
\hline $\mathrm{C}(6)-\mathrm{H}(6 \mathrm{~B})$ & 0.9800 & $\mathrm{H}(6 \mathrm{~A})-\mathrm{C}(6)-\mathrm{H}(6 \mathrm{~B})$ & 109.5 \\
\hline $\mathrm{C}(6)-\mathrm{H}(6 \mathrm{C})$ & 0.9800 & $\mathrm{O}(2)-\mathrm{C}(6)-\mathrm{H}(6 \mathrm{C})$ & 109.5 \\
\hline $\mathrm{C}(7)-\mathrm{H}(7 \mathrm{~A})$ & 0.9800 & $\mathrm{H}(6 \mathrm{~A})-\mathrm{C}(6)-\mathrm{H}(6 \mathrm{C})$ & 109.5 \\
\hline $\mathrm{C}(7)-\mathrm{H}(7 \mathrm{~B})$ & 0.9800 & $\mathrm{H}(6 \mathrm{~B})-\mathrm{C}(6)-\mathrm{H}(6 \mathrm{C})$ & 109.5 \\
\hline $\mathrm{C}(7)-\mathrm{H}(7 \mathrm{C})$ & 0.9800 & $\mathrm{O}(3)-\mathrm{C}(7)-\mathrm{H}(7 \mathrm{~A})$ & 109.5 \\
\hline $\mathrm{C}(8)-\mathrm{H}(8 \mathrm{~A})$ & 0.9800 & $\mathrm{O}(3)-\mathrm{C}(7)-\mathrm{H}(7 \mathrm{~B})$ & 109.5 \\
\hline $\mathrm{C}(8)-\mathrm{H}(8 \mathrm{~B})$ & 0.9800 & $\mathrm{H}(7 \mathrm{~A})-\mathrm{C}(7)-\mathrm{H}(7 \mathrm{~B})$ & 109.5 \\
\hline $\mathrm{C}(8)-\mathrm{H}(8 \mathrm{C})$ & 0.9800 & $\mathrm{O}(3)-\mathrm{C}(7)-\mathrm{H}(7 \mathrm{C})$ & 109.5 \\
\hline $\mathrm{C}(9)-\mathrm{H}(9 \mathrm{C})$ & 0.9800 & $\mathrm{H}(7 \mathrm{~A})-\mathrm{C}(7)-\mathrm{H}(7 \mathrm{C})$ & 109.5 \\
\hline $\mathrm{C}(9)-\mathrm{H}(9 \mathrm{~B})$ & 0.9800 & $\mathrm{H}(7 \mathrm{~B})-\mathrm{C}(7)-\mathrm{H}(7 \mathrm{C})$ & 109.5 \\
\hline$C(9)-H(9 C)$ & 0.9800 & $C(5)-C(8)-H(8 A)$ & 109.5 \\
\hline $\mathrm{C}(1)-\mathrm{O}(3)-\mathrm{C}(7)$ & $114.60(8)$ & $\mathrm{C}(5)-\mathrm{C}(8)-\mathrm{H}(8 \mathrm{~B})$ & 109.5 \\
\hline$C(1)-O(2)-C(6)$ & $114.62(8)$ & $\mathrm{H}(8 \mathrm{~A})-\mathrm{C}(8)-\mathrm{H}(8 \mathrm{~B})$ & 109.5 \\
\hline$C(3)-N(1)-C(5)$ & $122.57(9)$ & $\mathrm{C}(5)-\mathrm{C}(8)-\mathrm{H}(8 \mathrm{C})$ & 109.5 \\
\hline $\mathrm{C}(3)-\mathrm{N}(1)-\mathrm{H}(1)$ & 118.7 & $H(8 A)-C(8)-H(8 C)$ & 109.5 \\
\hline $\mathrm{C}(5)-\mathrm{N}(1)-\mathrm{H}(1)$ & 118.7 & $\mathrm{H}(8 \mathrm{~B})-\mathrm{C}(8)-\mathrm{H}(8 \mathrm{C})$ & 109.5 \\
\hline $\mathrm{O}(2)-\mathrm{C}(1)-\mathrm{O}(3)$ & $107.30(8)$ & $\mathrm{C}(5)-\mathrm{C}(9)-\mathrm{H}(9 \mathrm{~A})$ & 109.5 \\
\hline $\mathrm{O}(2)-\mathrm{C}(1)-\mathrm{C}(3)$ & $109.59(7)$ & & \\
\hline $\mathrm{O}(3)-\mathrm{C}(1)-\mathrm{C}(3)$ & $119.63(8)$ & & \\
\hline $\mathrm{O}(2)-\mathrm{C}(1)-\mathrm{C}(2)$ & $116.92(8)$ & & \\
\hline $\mathrm{O}(3)-\mathrm{C}(1)-\mathrm{C}(2)$ & $119.35(8)$ & & \\
\hline$C(3)-C(1)-C(2)$ & $82.51(7)$ & & \\
\hline
\end{tabular}


4. Torsion angles $\left[{ }^{\circ}\right]$

$\begin{array}{ll}\mathrm{C}(6)-\mathrm{O}(2)-\mathrm{C}(1)-\mathrm{O}(3) & 67.83(10) \\ \mathrm{C}(6)-\mathrm{O}(2)-\mathrm{C}(1)-\mathrm{C}(3) & -160.84(8) \\ \mathrm{C}(6)-\mathrm{O}(2)-\mathrm{C}(1)-\mathrm{C}(2) & -69.38(11) \\ \mathrm{C}(7)-\mathrm{O}(3)-\mathrm{C}(1)-\mathrm{O}(2) & -175.72(8) \\ \mathrm{C}(7)-\mathrm{O}(3)-\mathrm{C}(1)-\mathrm{C}(3) & 58.75(12) \\ \mathrm{C}(7)-\mathrm{O}(3)-\mathrm{C}(1)-\mathrm{C}(2) & -39.74(12) \\ \mathrm{O}(2)-\mathrm{C}(1)-\mathrm{C}(2)-\mathrm{O}(1) & 73.17(14) \\ \mathrm{O}(3)-\mathrm{C}(1)-\mathrm{C}(2)-\mathrm{O}(1) & -58.75(15) \\ \mathrm{C}(3)-\mathrm{C}(1)-\mathrm{C}(2)-\mathrm{O}(1) & -178.62(12) \\ \mathrm{O}(2)-\mathrm{C}(1)-\mathrm{C}(2)-\mathrm{C}(4) & -106.76(9) \\ \mathrm{O}(3)-\mathrm{C}(1)-\mathrm{C}(2)-\mathrm{C}(4) & 121.32(9) \\ \mathrm{C}(3)-\mathrm{C}(1)-\mathrm{C}(2)-\mathrm{C}(4) & 1.45(7) \\ \mathrm{C}(5)-\mathrm{N}(1)-\mathrm{C}(3)-\mathrm{C}(4) & -4.75(18) \\ \mathrm{C}(5)-\mathrm{N}(1)-\mathrm{C}(3)-\mathrm{C}(1) & 173.33(9)\end{array}$

$\mathrm{O}(2)-\mathrm{C}(1)-\mathrm{C}(3)-\mathrm{N}(1) \quad-64.05(13)$

$\mathrm{O}(3)-\mathrm{C}(1)-\mathrm{C}(3)-\mathrm{N}(1) \quad 60.38(14)$

$\mathrm{C}(2)-\mathrm{C}(1)-\mathrm{C}(3)-\mathrm{N}(1) \quad 179.98(11)$

$\mathrm{O}(2)-\mathrm{C}(1)-\mathrm{C}(3)-\mathrm{C}(4) \quad 114.48(9)$

$\mathrm{O}(3)-\mathrm{C}(1)-\mathrm{C}(3)-\mathrm{C}(4) \quad-121.09(9)$

$\mathrm{C}(2)-\mathrm{C}(1)-\mathrm{C}(3)-\mathrm{C}(4) \quad-1.49(7)$

$\mathrm{N}(1)-\mathrm{C}(3)-\mathrm{C}(4)-\mathrm{C}(2) \quad-179.99(12)$

$\mathrm{C}(1)-\mathrm{C}(3)-\mathrm{C}(4)-\mathrm{C}(2)$

$\mathrm{O}(1)-\mathrm{C}(2)-\mathrm{C}(4)-\mathrm{C}(3) \quad 178.50(13)$

$\mathrm{C}(1)-\mathrm{C}(2)-\mathrm{C}(4)-\mathrm{C}(3) \quad-1.58(8)$

$\mathrm{C}(3)-\mathrm{N}(1)-\mathrm{C}(5)-\mathrm{C}(8) \quad 123.60(10)$

$\mathrm{C}(3)-\mathrm{N}(1)-\mathrm{C}(5)-\mathrm{C}(9) \quad-112.26(11)$ 
IV. Copies of ${ }^{1} \mathrm{H}$ and ${ }^{13} \mathrm{C}$ NMR of known compounds $1,17 \mathbf{a}, 17 \mathbf{b}, \mathbf{1 7}, \mathbf{c}, \mathbf{1 7}, \mathbf{3 0 e}$

${ }^{1} \mathrm{H}$ NMR of 4-hydroxy-3-phenyl-3-cyclobutene-1,2-dione 1

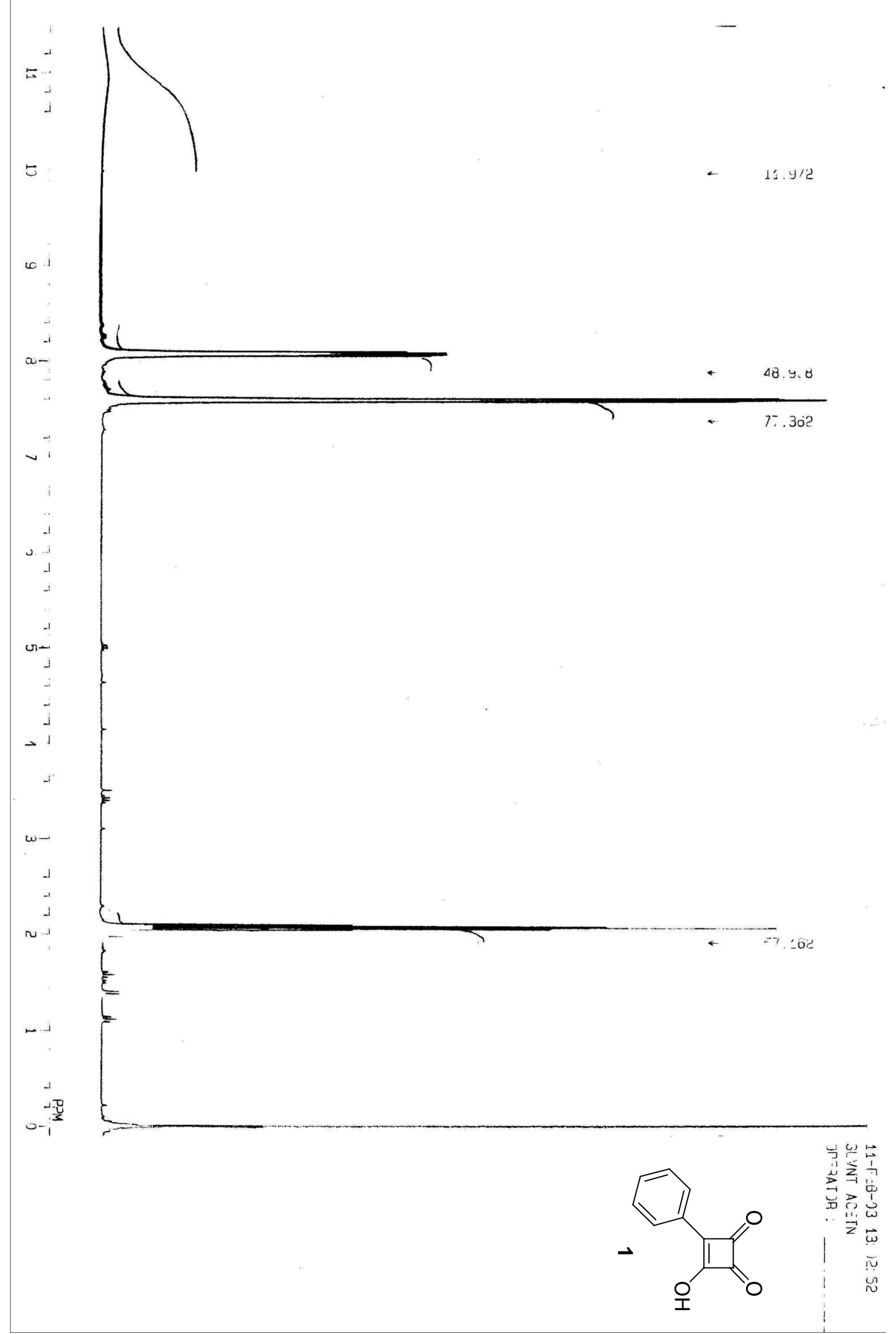


${ }^{13} \mathrm{C}$ NMR of 4-hydroxy-3-phenyl-3-cyclobutene-1,2-dione 1 
-S21-

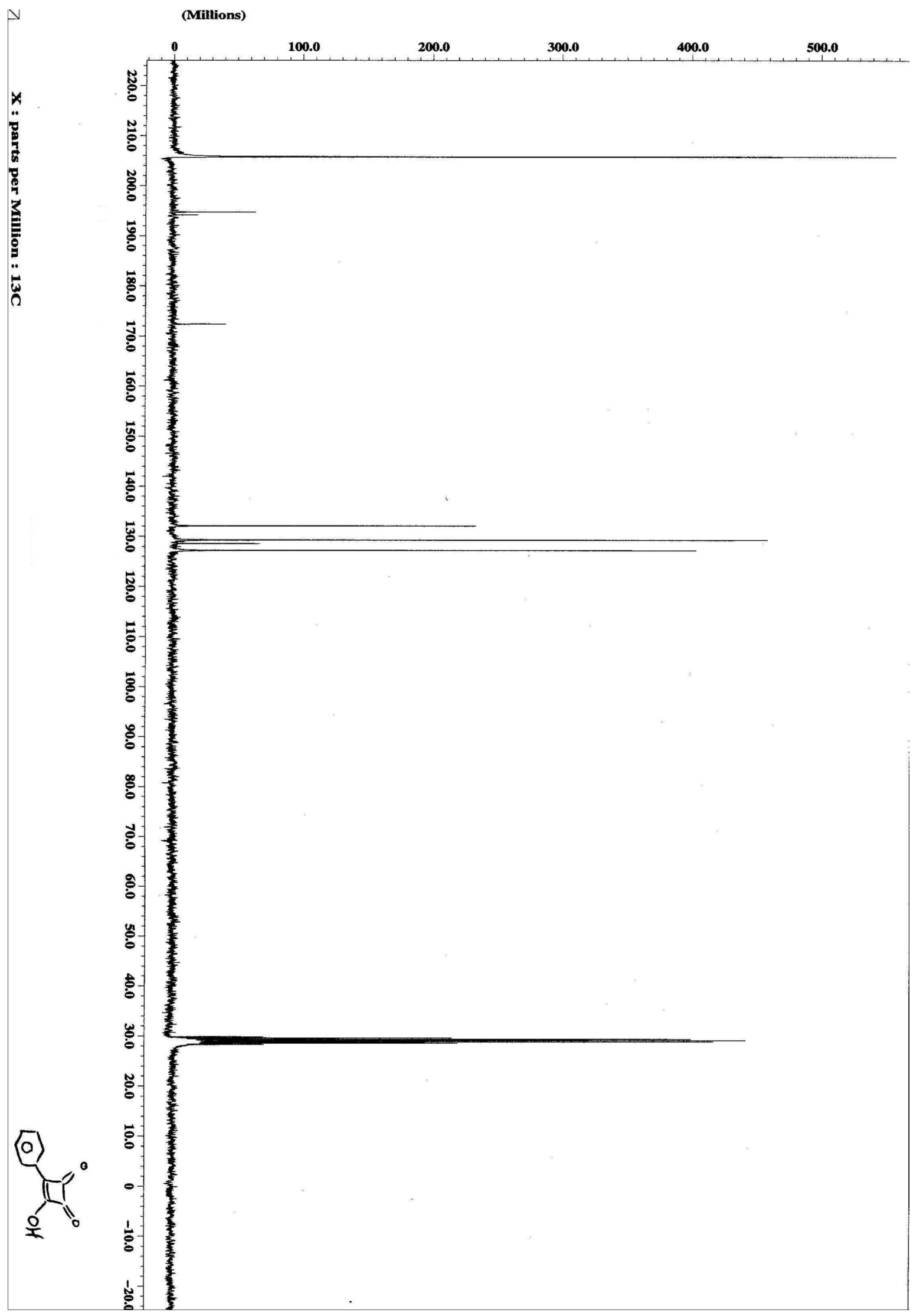




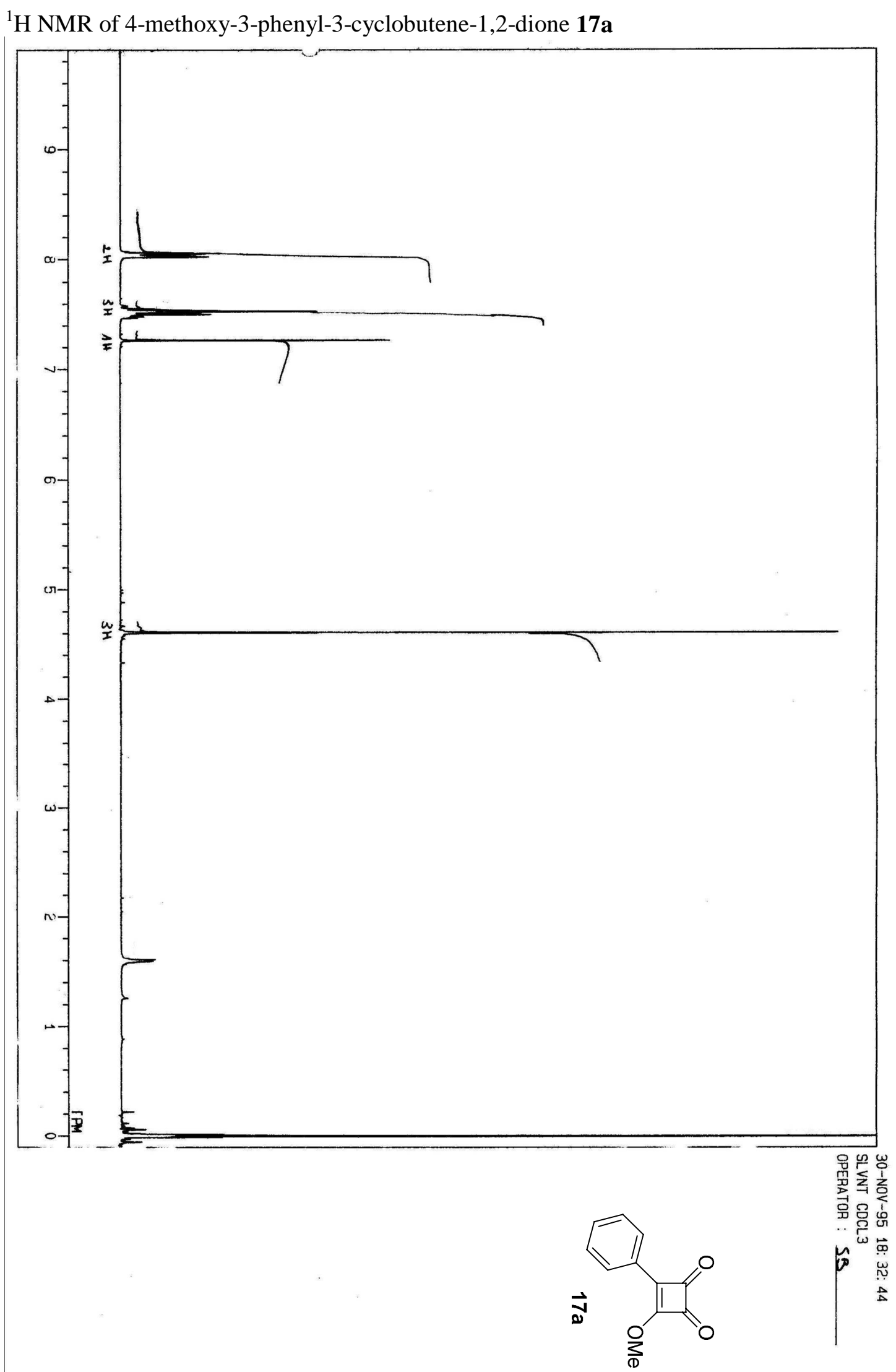


${ }^{13} \mathrm{C}$ NMR of 4-methoxy-3-phenyl-3-cyclobutene-1,2-dione 17a

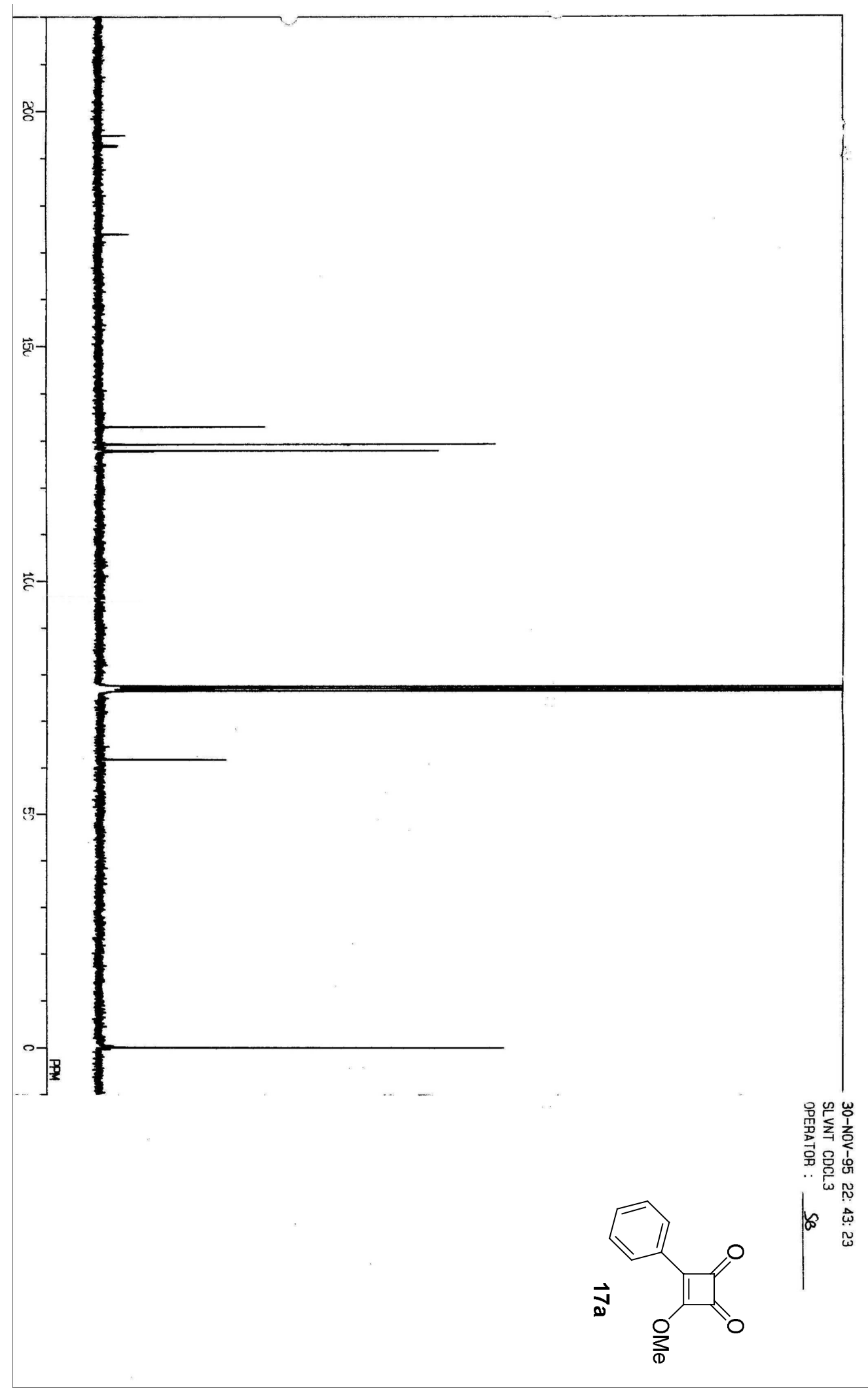




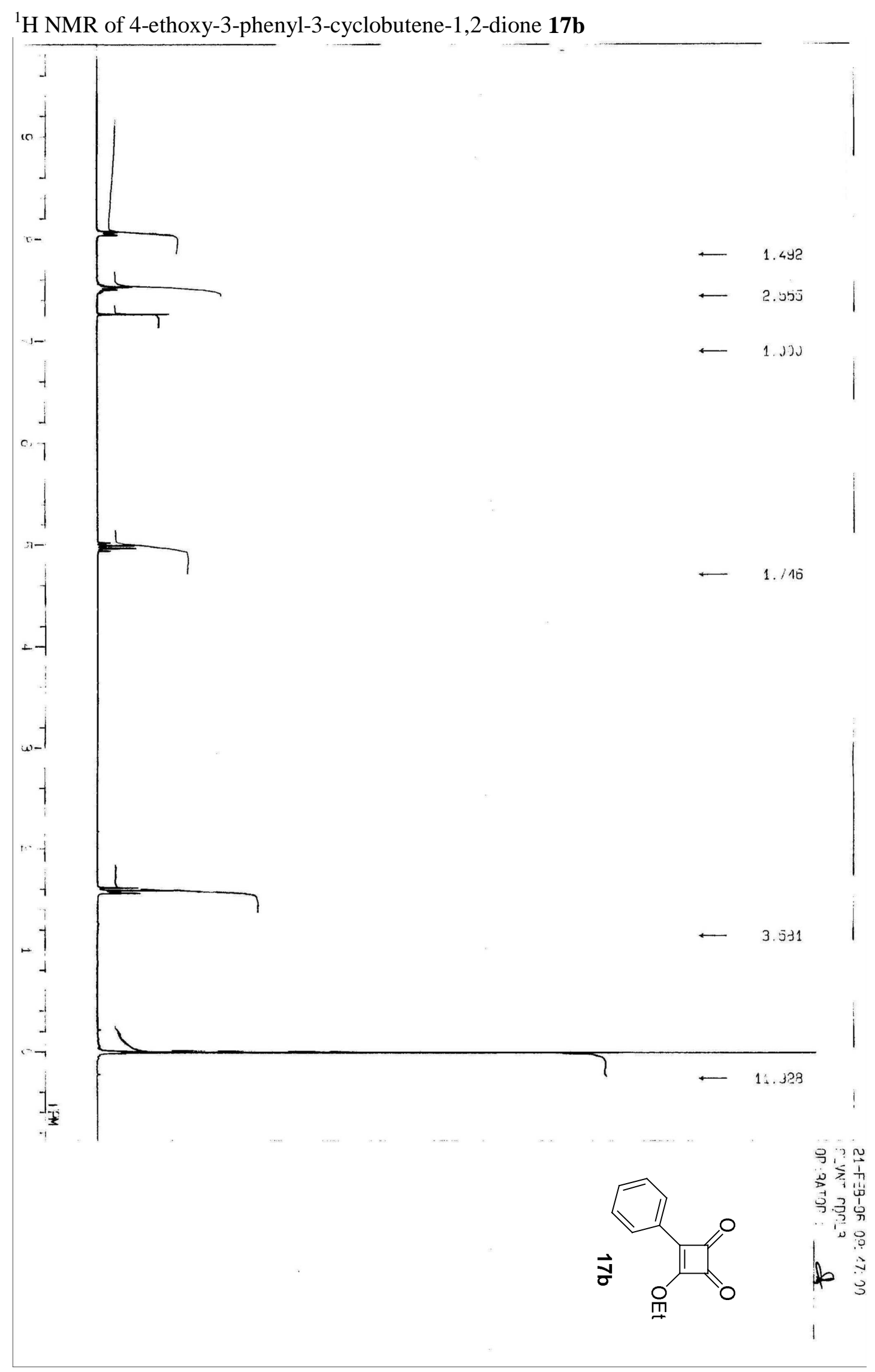


${ }^{13} \mathrm{C}$ NMR of 4-ethoxy-3-phenyl-3-cyclobutene-1,2-dione 17b

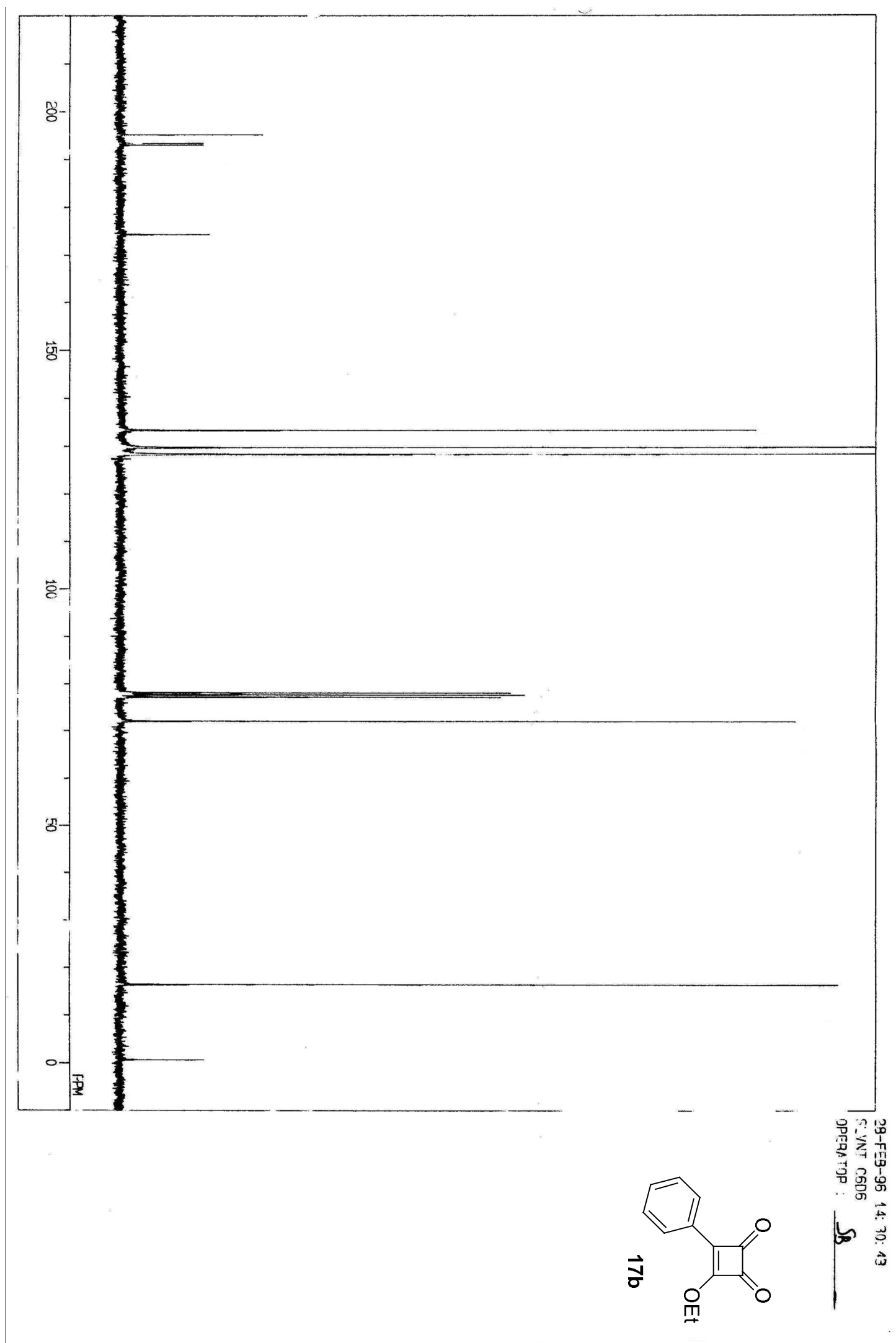


${ }^{1} \mathrm{H}$ NMR of 4-isopropoxy-3-phenyl-3-cyclobutene-1,2-dione 17c

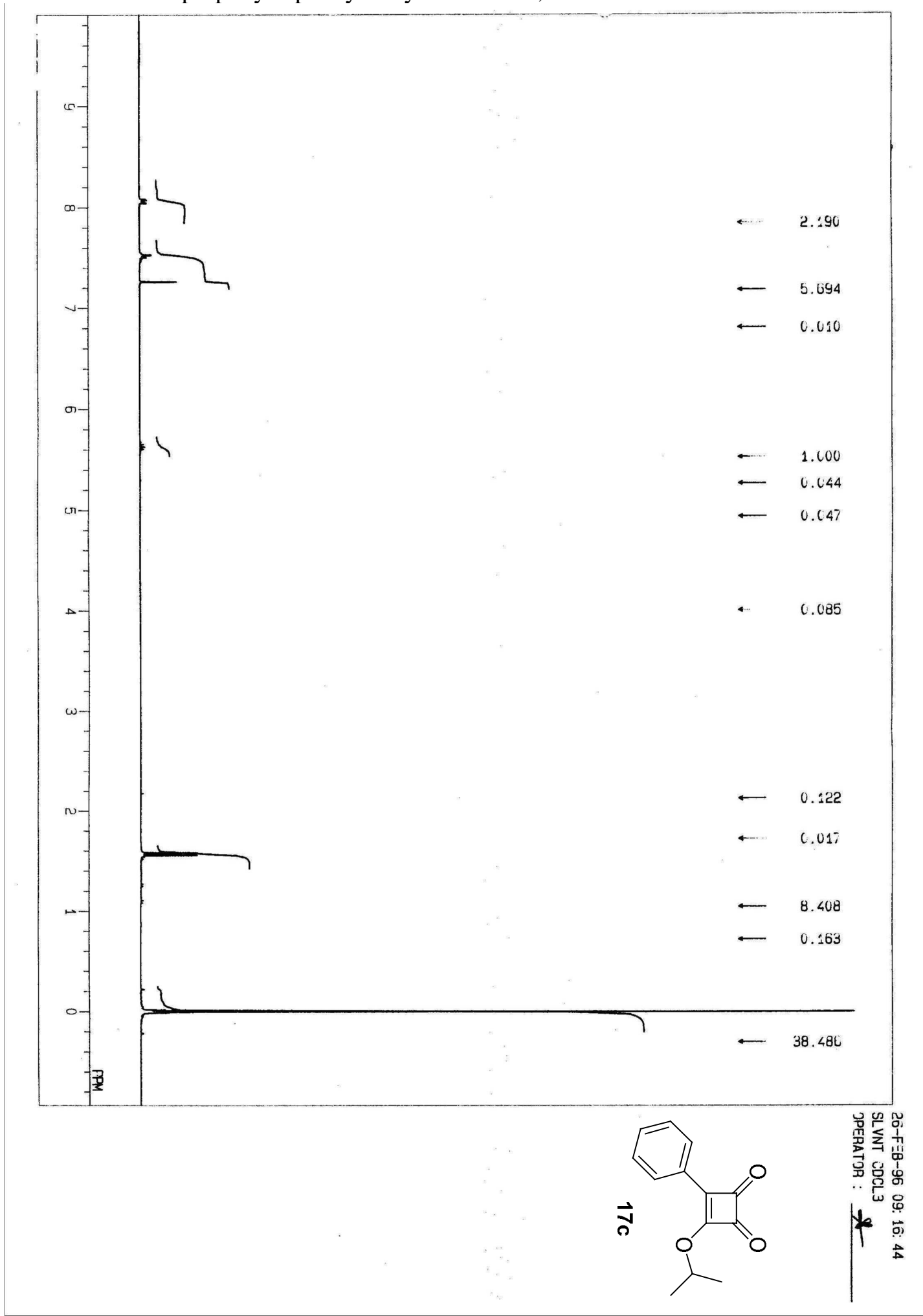




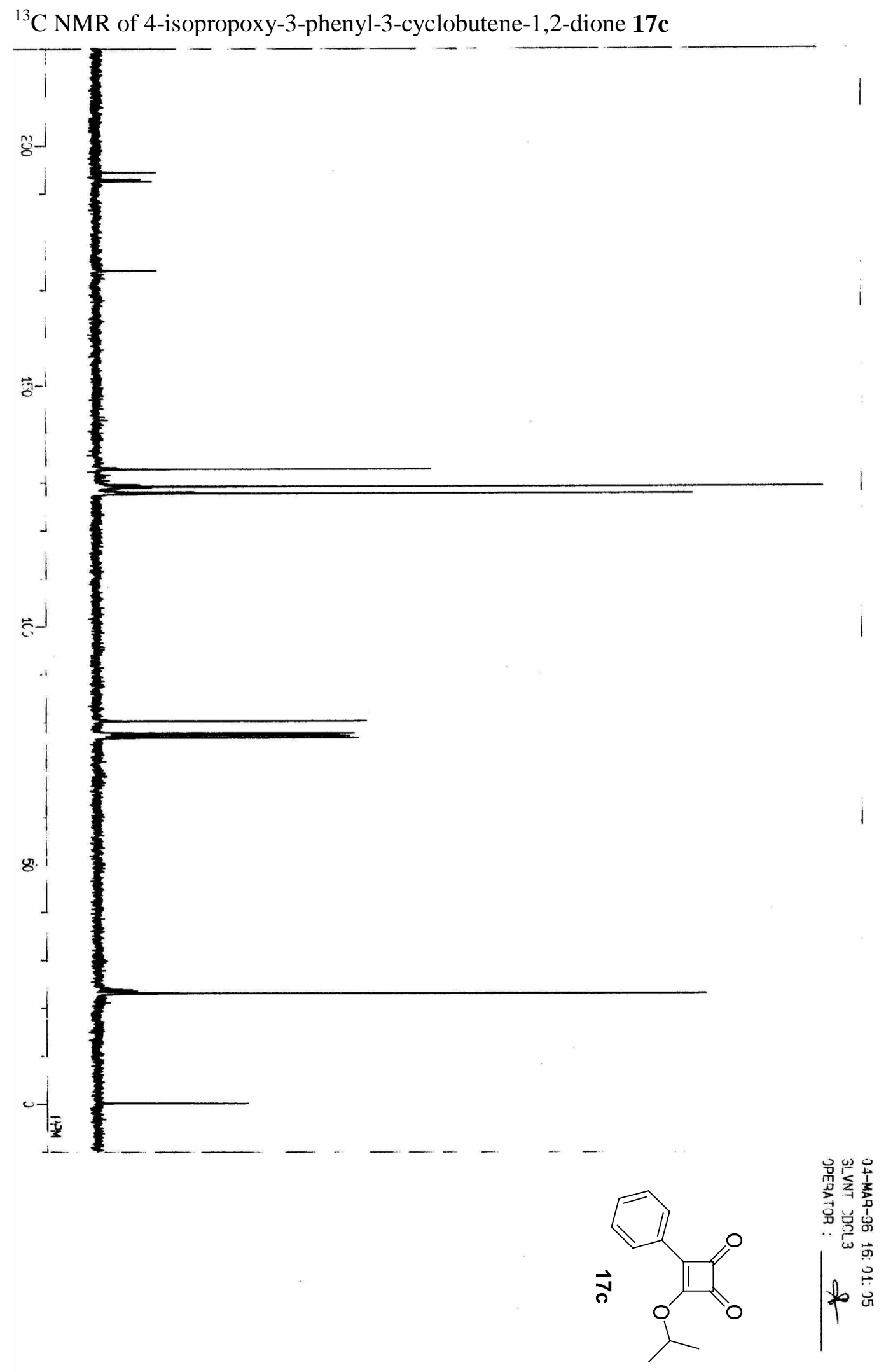




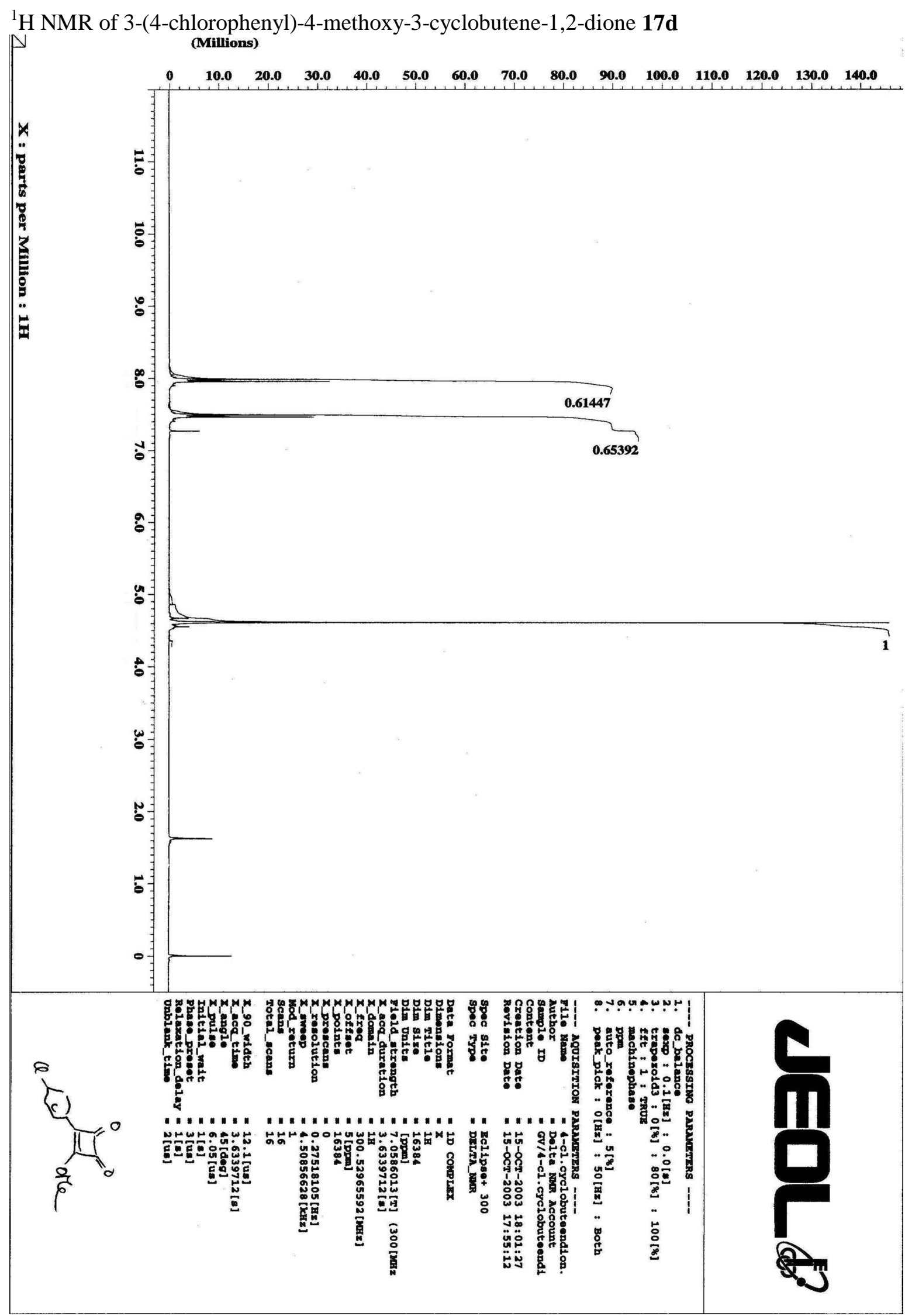


${ }^{13}$ C NMR of 3-(4-chlorophenyl)-4-methoxy-3-cyclobutene-1,2-dione 17d 


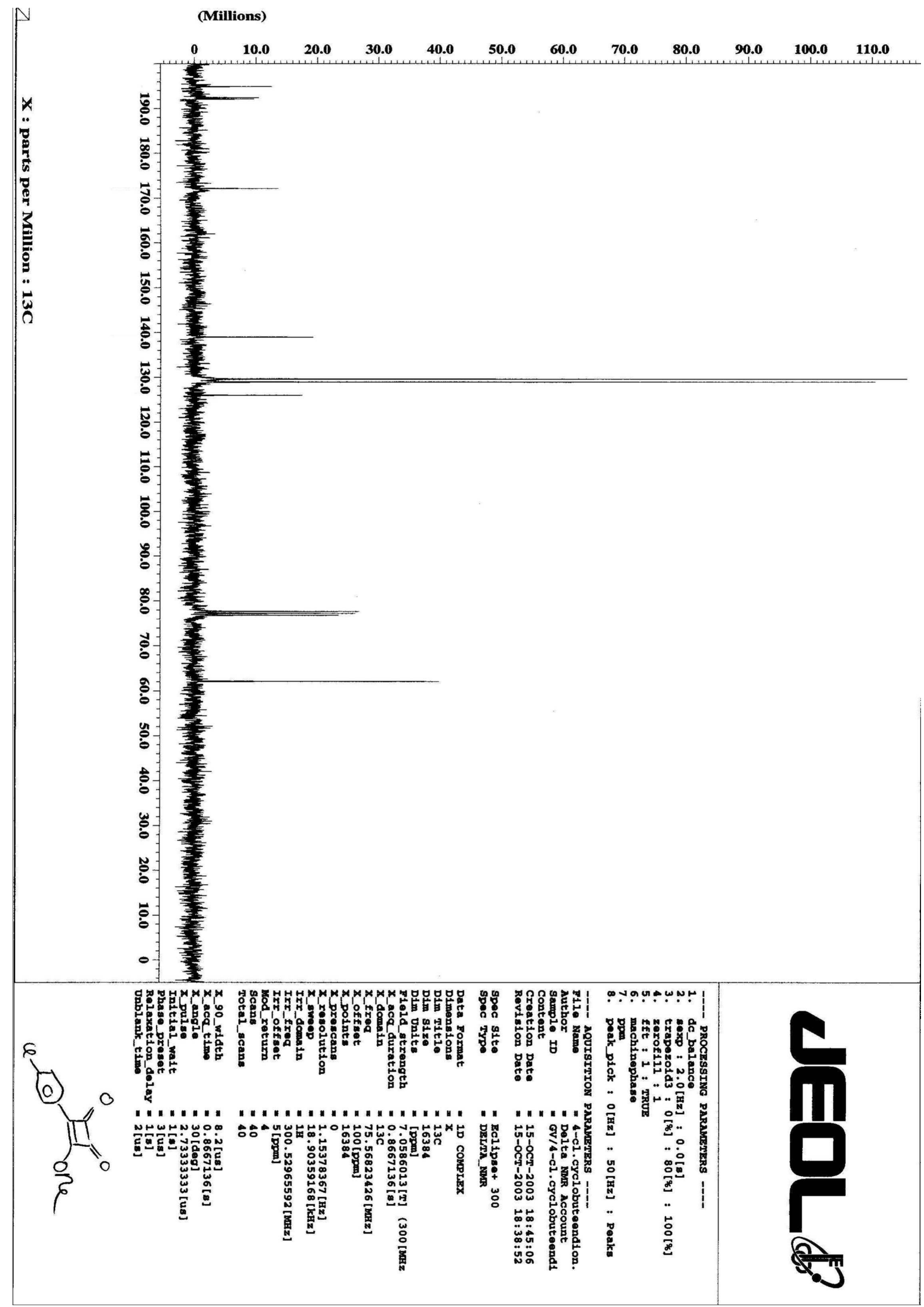

${ }^{1} \mathrm{H}$ NMR of 3-t-butylamino-3-cyclobutene-1,2-dione 30e 


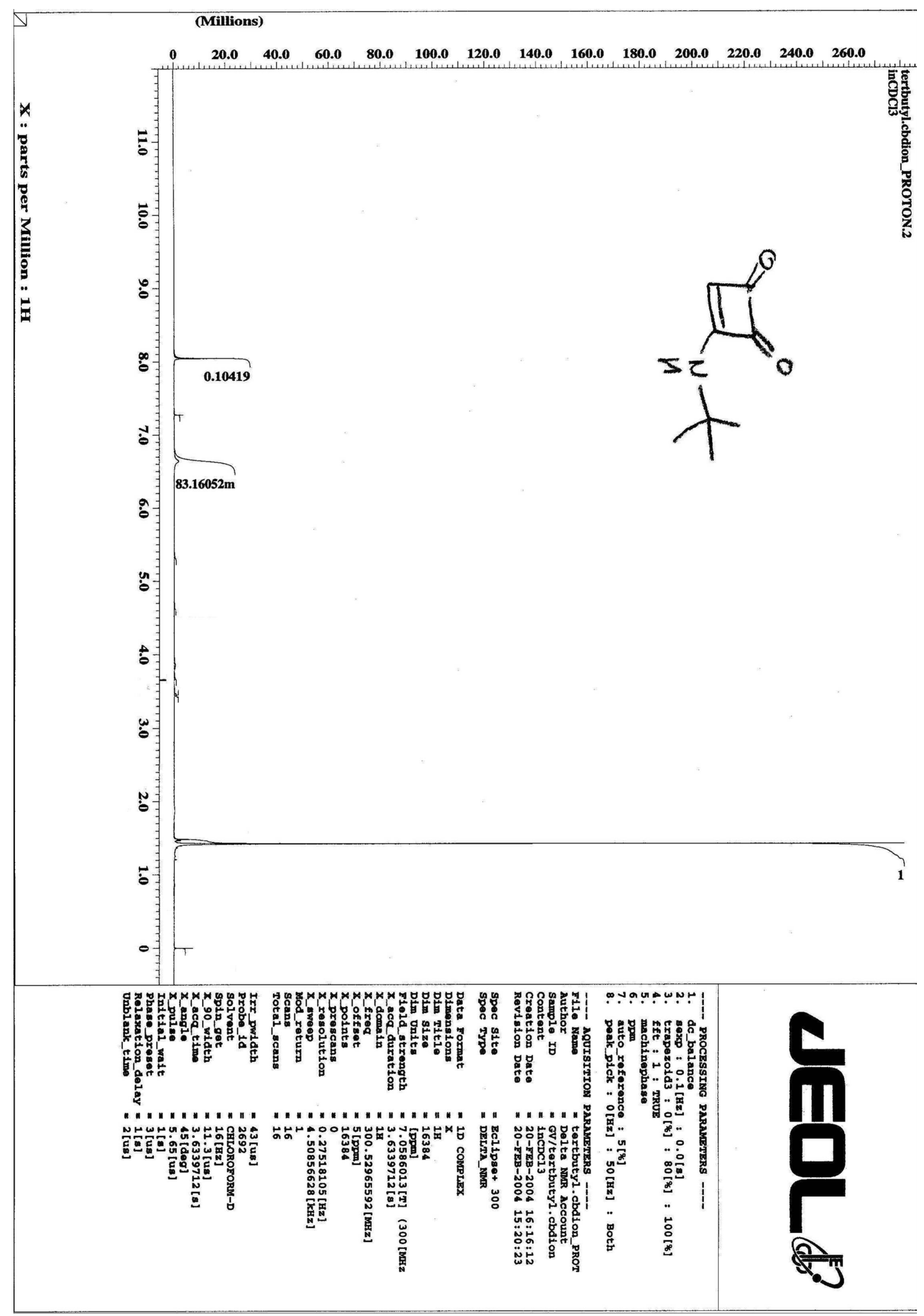

${ }^{13} \mathrm{C}$ NMR of 3-t-butylamino-3-cyclobutene-1,2-dione 30e 


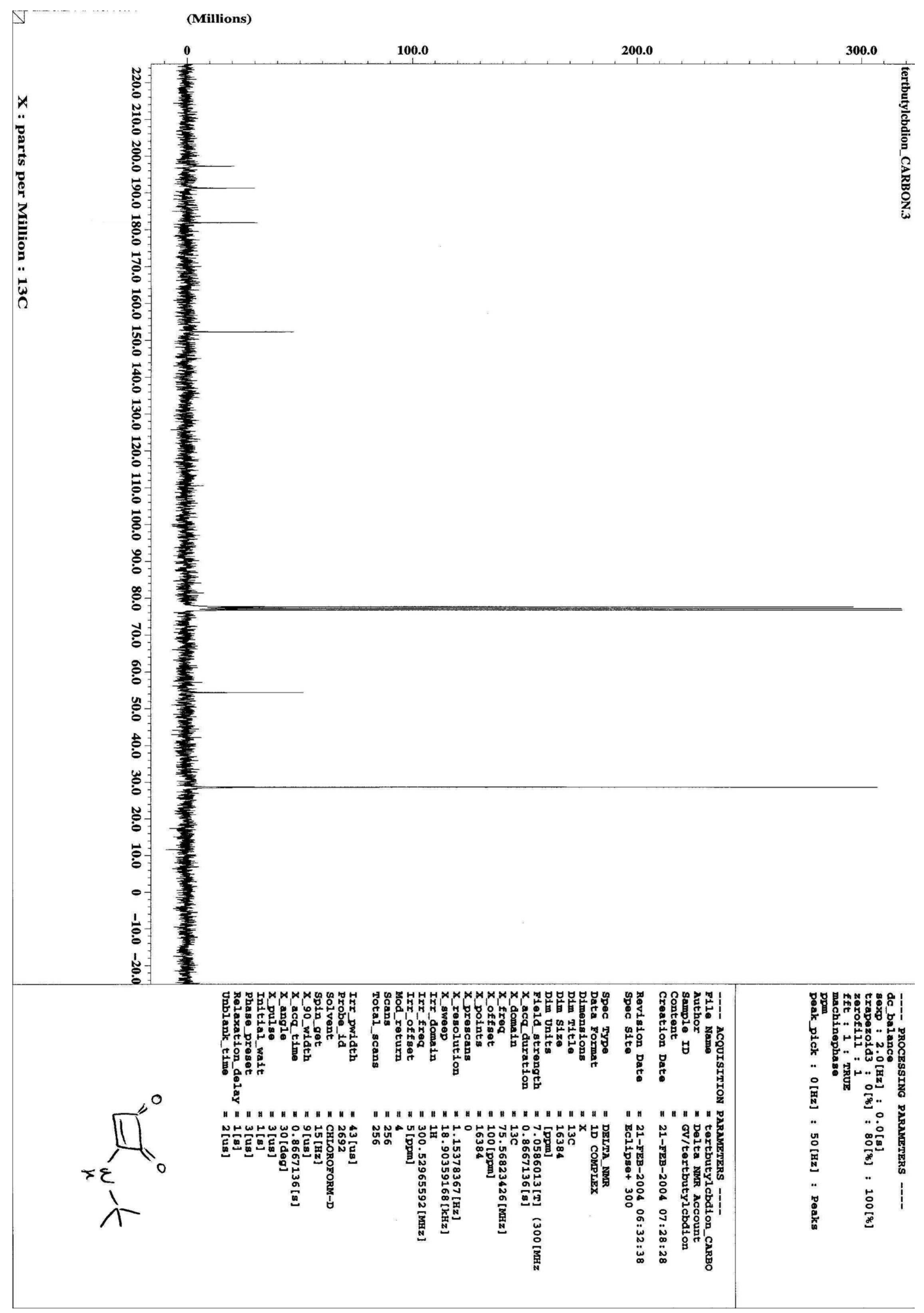

